TRANSACTIONS OF THE

AMERICAN MATHEMATICAL SOCIETY

Volume 365, Number 7, July 2013, Pages 3373-3396

S 0002-9947(2012)05625-X

Article electronically published on December 3, 2012

\title{
INEQUALITIES BETWEEN THE CHERN NUMBERS OF A SINGULAR FIBER IN A FAMILY OF ALGEBRAIC CURVES
}

\author{
JUN LU AND SHENG-LI TAN
}

\begin{abstract}
In a family of curves, the Chern numbers of a singular fiber are the local contributions to the Chern numbers of the total space. We will give some inequalities between the Chern numbers of a singular fiber as well as their lower and upper bounds. We introduce the dual fiber of a singular fiber, and prove a duality theorem. As an application, we will classify singular fibers with large or small Chern numbers.
\end{abstract}

\section{INTRODUCTION AND MAIN RESULTS}

Chern numbers of a singular fiber in a family of curves are the local contributions of the fiber to the global Chern numbers of the total space. The first purpose of this paper is to find the best inequalities between the Chern numbers of a singular fiber. Our second purpose is to try to give a new approach to the classification of singular fibers of genus $g$. We know that when $g$ is large, there are too many singular fibers of genus $g$ to classify completely (see [6, 8, [9, 17]). Some authors are trying to find a new method to classify singular fibers of small genus (see, for example, 2]). In order to get the local-global relations between the invariants, one possible way is to classify singular fibers according to their contributions to the global invariants. To explain this approach, we will classify singular fibers with large or small Chern numbers and give some applications. See the survey [3] for the background of the study on the local-global properties for families of curves.

A family of curves of genus $g$ over $C$ is a fibration $f: X \rightarrow C$ whose general fibers $F$ are smooth curves of genus $g$, where $X$ is a complex smooth projective surface. The family is called semistable if all of the singular fibers are reduced nodal curves. If $X=F \times C$ and $f$ is just the second projection to $C$, then we call $f$ a trivial family. If all of the smooth fibers of $f$ are isomorphic to each other, equivalently, $f$ becomes trivial under a finite base change $\tilde{C} \rightarrow C$, then $f$ is called isotrivial. We always assume that $f$ is relatively minimal, i.e., there is no $(-1)$-curve in any singular fiber.

When $g=1$, Kodaira [7] found the global invariants from the singular fibers. The first Chern number $c_{1}^{2}(X)$ is always zero, the second Chern number $c_{2}(X)$ is

Received by the editors March 7, 2010 and, in revised form, March 19, 2011.

2010 Mathematics Subject Classification. Primary 14D06, 14C21, $14 \mathrm{H} 10$.

Key words and phrases. Chern number, singular fiber, modular invariant, isotrivial, classification.

This work was supported by NSFC, the Science Foundations of the Education Ministry of China and the Foundation of Scientific Program of Shanghai.

(C) 2012 American Mathematical Society Reverts to public domain 28 years from publication 
equal to $12 \chi\left(\mathcal{O}_{X}\right)$ by Noether's formula, and

$$
\text { (1.1) } c_{2}(X)=j+6 \nu\left(\mathrm{I}^{*}\right)+2 \nu(\mathrm{II})+10 \nu\left(\mathrm{II}^{*}\right)+3 \nu(\mathrm{III})+9 \nu\left(\mathrm{III}^{*}\right)+4 \nu(\mathrm{IV})+8 \nu\left(\mathrm{IV}^{*}\right),
$$

where $\nu(\mathrm{T})$ denotes the number of singular fibers of type $\mathrm{T}$, and $j$ is the number of poles of the $J$-function of the family. Note that the $J$-function over $C$ induces a holomorphic map of degree $j$ from $C$ to the moduli space $\overline{\mathcal{M}}_{1}$ of elliptic curves. So $j$ depends only on the generic fibers.

By introducing the Chern numbers $c_{1}^{2}(F), c_{2}(F)$ and $\chi_{F}$ for a singular fiber $F$, the second author ([13, [14], 15]) generalized Kodaira's formula (1.1) to the higher genus case,

$$
\left\{\begin{array}{l}
c_{1}^{2}(X)=\kappa(f)+8(g-1)(g(C)-1)+\sum_{i=1}^{s} c_{1}^{2}\left(F_{i}\right), \\
c_{2}(X)=\delta(f)+4(g-1)(g(C)-1)+\sum_{i=1}^{s} c_{2}\left(F_{i}\right), \\
\chi\left(\mathcal{O}_{X}\right)=\lambda(f)+(g-1)(g(C)-1)+\sum_{i=1}^{s} \chi_{F_{i}},
\end{array}\right.
$$

where $F_{1}, \cdots, F_{s}$ are all singular fibers of $f$, and $\kappa(f), \delta(f)$ and $\lambda(f)$ are the modular invariants of the family. $f$ also induces a holomorphic map from $C$ to the moduli space of semistable curves of genus $g$ :

$$
J: C \longrightarrow \overline{\mathcal{M}}_{g} \text {. }
$$

Then $\kappa(f)=\operatorname{deg} J^{*} \kappa, \delta(f)=\operatorname{deg} J^{*} \delta$ and $\lambda(f)=\operatorname{deg} J^{*} \lambda$, where $\lambda, \delta$ and $\kappa$ are respectively the Hodge divisor class, the boundary divisor class and $\kappa=12 \lambda-\delta$. In the case of elliptic fibrations, $\kappa(f)=0$ and $\delta(f)=j$.

Let $\widetilde{f}: \widetilde{X} \rightarrow \widetilde{C}$ be a semistable reduction of $F$ under any base change $\pi: \widetilde{C} \rightarrow C$ ramified over $p=f(F)$ and some non-critical points of $f$. The Chern numbers of $F$ are defined as follows:

$$
c_{1}^{2}(F)=K_{f}^{2}-\frac{1}{d} K_{\widetilde{f}}^{2}, \quad c_{2}(F)=e_{f}-\frac{1}{d} e_{\tilde{f}}, \quad \chi_{F}=\chi_{f}-\frac{1}{d} \chi_{\tilde{f}},
$$

where $d$ is the degree of $\pi$, and $K_{f}^{2}=c_{1}^{2}(X)-8(g-1)(g(C)-1), e_{f}=c_{2}(X)$ $-4(g-1)(g(C)-1)$ and $\chi_{f}=\chi\left(\mathcal{O}_{X}\right)-(g-1)(g(C)-1)$ are the relative invariants of $f$. These Chern numbers are independent of the choice of the semistable reductions $\pi$. If $g=1$, then $c_{1}^{2}(F)=0$ and $c_{2}(F)$ is exactly the coefficient in (1.1) according to the type of the fiber $F$. See 33.3 for the computation formulas for the Chern numbers of $F$. We briefly summarize the known properties of the Chern numbers. Assume that $g=g(F) \geq 2$ and $F$ contains no $(-1)$-curves.

(1) Positivity: $c_{1}^{2}(F), c_{2}(F)$ and $\chi_{F}$ are non-negative rational numbers, and one of the three numbers vanishes if and only if $F$ is semistable.

(2) Noether's equality: $c_{1}^{2}(F)+c_{2}(F)=12 \chi_{F}$.

(3) Blow-up formulas: $c_{1}^{2}\left(F^{\prime}\right)=c_{1}^{2}(F)-1, c_{2}\left(F^{\prime}\right)=c_{2}(F)+1, \chi_{F^{\prime}}=\chi_{F}$, where $F^{\prime}=\sigma^{*} F$ is the pullback of $F$ under the blowing up $\sigma: X^{\prime} \rightarrow X$ at a point $p$ on $F$.

(4) Canonical class inequality: $c_{1}^{2}(F) \leq 4 g-4$.

(5) Miyaoka-Yau type inequality: $c_{1}^{2}(F) \leq 2 c_{2}(F)$ or, equivalently, $c_{1}^{2}(F) \leq$ $8 \chi_{F}$, with equality iff $F_{\text {red }}$ is a nodal curve and $F=n F_{\text {red }}$ for some positive integer $n$.

The positivity is essentially due to Beauville [4, Xiao [19] and the second author [11. Noether's equality and the blow-up formulas are direct consequences of the definition of Chern numbers. The last two inequalities can be found in [13. 
Let $\bar{F}$ be the normal crossing fiber obtained by blowing up the singularities of $F(\bar{F}$ is called the normal crossing model of $F)$. Write $\bar{F}=n_{1} C_{1}+\cdots+n_{k} C_{k}$, where the $C_{i}$ 's are the irreducible components. Denote by $M_{F}$ the least common multiplicity of $n_{1}, \cdots, n_{k}$. Let $n$ be a positive integer satisfying $n \equiv-1\left(\bmod M_{F}\right)$. Denote by $F^{*}$ the fiber obtained from $F$ by a local base change $\pi$ defined by $w=z^{n}$. We call $F^{*}$ the dual fiber of $F$ (see $\S 2$ ). This is a natural generalization of Kodaira's dual fibers for elliptic fibrations. Our first result is the duality theorem for $\chi_{F}$.

Theorem 1.1 (Duality theorem for $\chi$ ). Let $\bar{F}$ and $\bar{F}^{*}$ be the normal crossing models of $F$ and $F^{*}$ respectively. Let $N_{\bar{F}}=g-p_{a}\left(\bar{F}_{\text {red }}\right)$. Then $0 \leq N_{\bar{F}} \leq g$.

1) $N_{\bar{F}}=N_{\bar{F}^{*}}$, i.e., $p_{a}\left(\bar{F}_{\text {red }}\right)=p_{a}\left(\bar{F}_{\text {red }}^{*}\right)$.

2) $\chi_{F}+\chi_{F^{*}}=N_{\bar{F}}$.

3) $\frac{1}{6} N_{\bar{F}} \leq \chi_{F} \leq \frac{5}{6} N_{\bar{F}} \cdot \chi_{F}=\frac{1}{6} N_{\bar{F}}$ (resp. $\frac{5}{6} N_{\bar{F}}$ ) if and only if $F\left(\right.$ resp. $F^{*}$ ) is a reduced curve whose singularities are at worst ordinary cusps or nodes.

In general, $F^{* *}$ is not necessarily equal to $F$, but we have the equality $\chi_{F^{* *}}=\chi_{F}$.

Theorem 1.2. Assume that $g \geq 2$. We have the following optimal inequalities.

1) If $F$ is not semistable, then $c_{2}(F) \geq \frac{11}{6}$ and $\chi_{F} \geq \frac{1}{6}$. One of the equalities holds if and only if $F$ is a reduced curve with one ordinary cusp and some nodes.

2) $c_{1}^{2}(F) \leq 4 g-\frac{24}{5}$. More precisely, if $g \geq 7$ or $g=5$, then $c_{1}^{2}(F) \leq 4 g-\frac{11}{2}$.

$$
c_{1}^{2}(F) \leq \begin{cases}\frac{16}{5}, & g=2, \\ 7, & g=3, \\ \frac{54}{5}, & g=4, \\ \frac{130}{7}, & g=6 .\end{cases}
$$

3) (Arakelov type inequality) $\chi_{F} \leq \frac{5 g}{6}$, with equality iff $F^{*}$ is a reduced curve with nodes and ordinary cusps as its singularities, and its normal crossing model is a tree of smooth rational curves. 5.1).

For any $g \geq 2$, there is a numerical fiber $F$ with $c_{1}^{2}(F)=4 g-\frac{11}{2}$ (see Example

Theorem 1.3. Let $F$ be a minimal singular fiber of genus $g \geq 2$ satisfying $c_{1}^{2}(F)>$ $4 g-\frac{11}{2}$. Then $g \leq 6$ and $F$ is one of the following 22 fibers. $\circ$ is a (-2)-curve, and - is a (-3)-curve.
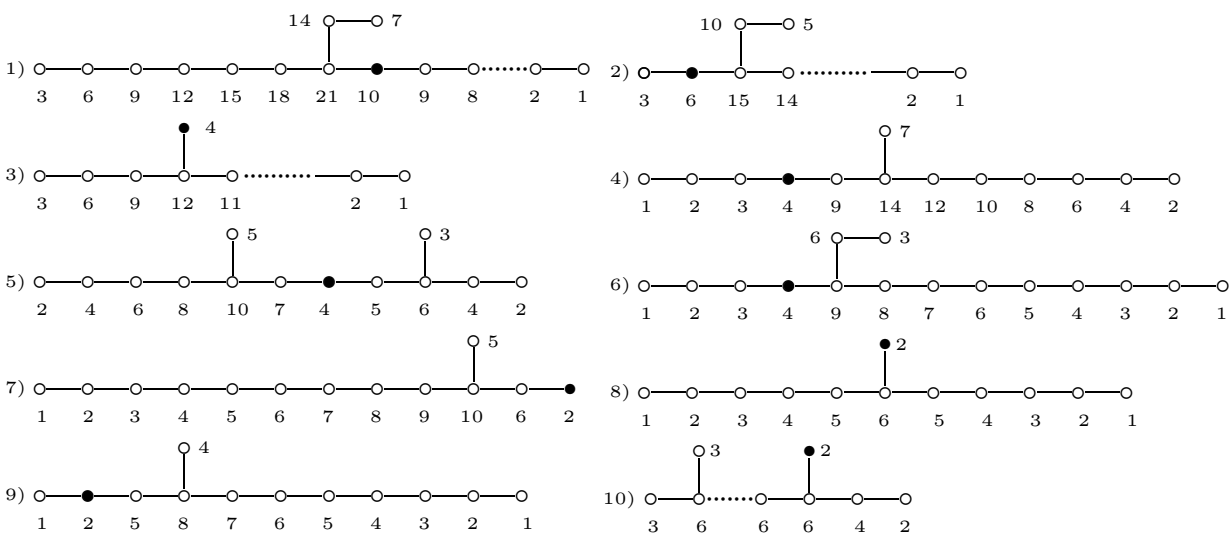

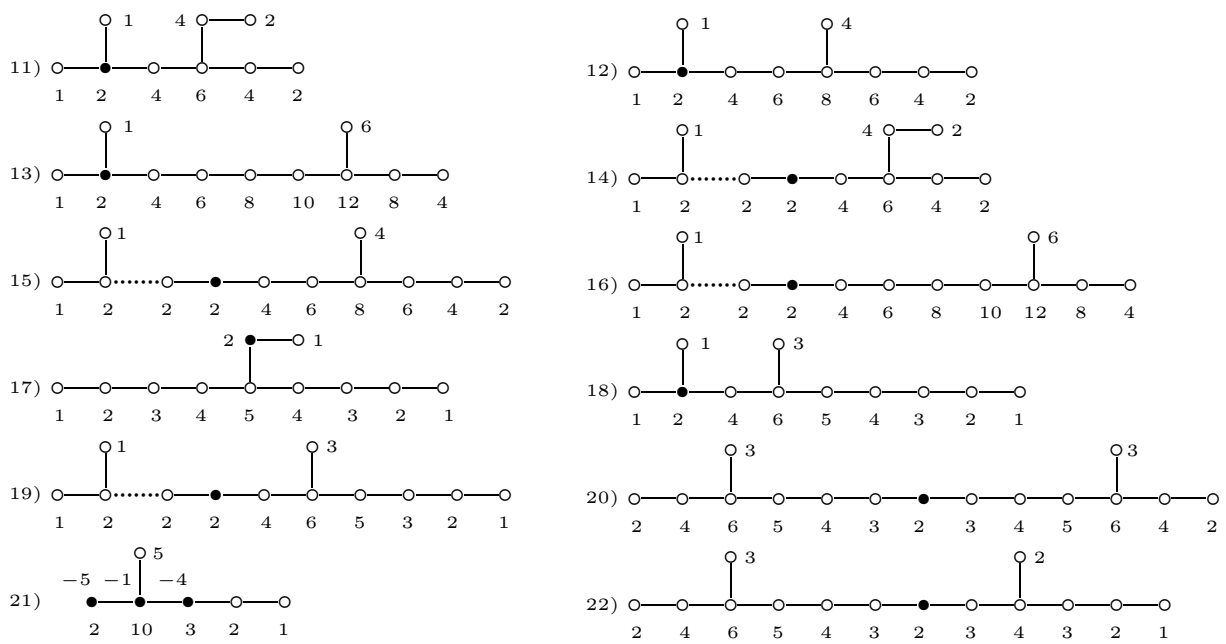

See $\S 5.5$ for the Chern numbers of these 22 fibers.

Theorem 1.4. Assume that $g \geq 2$. If $2 c_{2}(F)-c_{1}^{2}(F)<6$, then either $F=n C$ for some smooth curve $C$ or $F_{\text {red }}$ admits at most one singular point $p$ other than nodes. One of the following cases occurs.

I) $F=n F_{\text {red }}$.

1) $F_{\text {red }}$ is a smooth or nodal curve.

2) $p$ is of type $A_{2}$.

3) $p$ is of type $A_{3}$ and any (-2)-curve does not pass through $p$.

4) $p$ is of type $A_{3}$ and one (-2)-curve passes through $p$.

5) $p$ is of type $D_{4}$.

II) $F=n A+2 n B$, where $A$ and $B$ are reduced nodal curves without common components, $A B=2, A^{2}=-4$ and $B^{2}=-1$. A has at most two connected components $A_{1}$ and $A_{2}$.

6) $A \cap B=\{p, q\}$ and any (-2)-curve is not a connected component of $A$.

7) A has two connected components and one is a (-2)-curve.

8) $A$ and $B$ are tangent at a point $p$.

The invariants of these fibers $F$ are as follows, where $0 \leq N=g(F)-p_{a}\left(F_{\text {red }}\right) \leq$ $g$.

\begin{tabular}{|c|c|c|c|c|c|c|c|c|}
\hline$F$ & 1 & 2 & 3 & 4 & 5 & 6 & 7 & 8 \\
\hline $2 c_{2}-c_{1}^{2}$ & 0 & $\frac{7}{2}$ & $\frac{9}{2}$ & $\frac{21}{4}$ & 5 & 3 & $\frac{9}{2}$ & $\frac{11}{2}$ \\
\hline$c_{1}^{2}-4 N$ & 0 & $\frac{1}{6}$ & $\frac{1}{2}$ & $\frac{1}{4}$ & 1 & -1 & $-\frac{3}{2}$ & $-\frac{1}{2}$ \\
\hline$c_{2}-2 N$ & 0 & $\frac{11}{6}$ & $\frac{5}{2}$ & $\frac{11}{4}$ & 3 & 1 & $\frac{3}{2}$ & $\frac{5}{2}$ \\
\hline$\chi-\frac{1}{2} N$ & 0 & $\frac{1}{6}$ & $\frac{1}{4}$ & $\frac{1}{4}$ & $\frac{1}{3}$ & 0 & 0 & $\frac{1}{6}$ \\
\hline
\end{tabular}

Note that $2 c_{2}-c_{1}^{2}<6$ is equivalent to $8 \chi-c_{1}^{2}<2$. Hence the fibers satisfying $c_{2}(F) \leq 3$ or $\chi \leq \frac{1}{4}$ are included in the classification list 1) $\sim 8$ ). For a nonsemistable fiber, $c_{1}^{2}, c_{2}$ and $\chi$ are positive. Therefore, one can check that $\frac{11}{6}$ (resp. 
$\frac{1}{6}$ ) is the lower bound of $c_{2}$ (resp. $\chi$ ) for non-semistable fibers. All of the fibers from 2) to 8) cannot be the fibers in an isotrivial family of curves because their semistable models are not smooth.

Corollary 1.5. Let $s$ be the number of singular fibers of $f: X \rightarrow C$ and $g \geq 2$.

1) If $f$ is non-trivial, then $\chi_{f} \leq \frac{g}{2}\left(2 g(C)-2+\frac{8}{3} s\right)$.

2) If $f$ is isotrivial, then $K_{f}^{2} \leq\left(4 g-\frac{24}{5}\right) s$, and $\chi_{f} \leq \frac{5 g s}{6}$.

As an application of Theorem 1.4, we have

Corollary 1.6. Assume $f: X \rightarrow C$ is isotrivial. Let $s$ be the number of singular fibers that are not multiples of a smooth curve. Then $K_{X}^{2} \leq 8 \chi\left(\mathcal{O}_{X}\right)-2 s$.

This gives a new proof of Polizzi's theorem that $K_{X}^{2} \neq 8 \chi\left(\mathcal{O}_{X}\right)-1$ when $f$ : $X \rightarrow C$ is isotrivial [10. We will give some other applications of the main results in each section.

\section{Dual Models $F^{*}$ OF A FiBer $F$}

We recall several models of a singular fiber in this section, including the minimal model, normal crossing model, $n$-th root model, semistable model, and the dual model.

2.1. Normal crossing model. A curve $B$ on $X$ is a non-zero effective divisor.

Definition 2.1. A partial resolution of the singularities of $B$ is a sequence of blowing-ups $\sigma=\sigma_{1} \circ \sigma_{2} \circ \cdots \circ \sigma_{r}: \bar{X} \rightarrow X$,

$$
\left(\bar{X}, \sigma^{*} B\right)=\left(X_{r}, B_{r}\right) \stackrel{\sigma_{r}}{\longrightarrow} X_{r-1} \stackrel{\sigma_{r-1}}{\longrightarrow} \cdots \stackrel{\sigma_{2}}{\longrightarrow}\left(X_{1}, B_{1}\right) \stackrel{\sigma_{1}}{\longrightarrow}\left(X_{0}, B_{0}\right)=(X, B),
$$

satisfying the following conditions:

(i) $B_{r, \text { red }}$ has at worst ordinary double points as its singularities.

(ii) $B_{i}=\sigma_{i}^{*} B_{i-1}$ is the total transform of $B_{i-1}$.

Furthermore, $\sigma$ is called the minimal partial resolution of the singularities of $B$ if

(iii) $\sigma_{i}$ is the blowing-up of $X_{i-1}$ at a singular point $\left(B_{i-1, \text { red }}, p_{i-1}\right)$ which is not an ordinary double point for any $i \leq r$.

The minimal model of $F$ is obtained by contracting all $(-1)$-curves in $F$. Denote by $\bar{F}$ the partial resolution of the singularities of the minimal model of $F$.

Definition 2.2. $\bar{F}$ is called the normal crossing model of $F$. If $\sigma$ is minimal, then we say that $\bar{F}$ is the minimal normal crossing model of $F$.

A $(-1)$-curve in $\bar{F}$ is called redundant if it meets the other components in at most two points. It is obvious that a redundant $(-1)$-curve can be contracted without introducing singularities worse than ordinary double points. The minimal normal crossing model of $F$ contains no redundant $(-1)$-curves, and it can be obtained from any normal crossing model by contracting all redundant $(-1)$-curves. In fact, the minimal normal crossing model of $F$ is determined uniquely by $F$. 
2.2. The $n$-th root model and the semistable model of $F$. Let $\pi: \widetilde{C} \rightarrow C$ be a base change of degree $n$. Then we can construct the pullback fibration $\widetilde{f}: \widetilde{X} \rightarrow \widetilde{C}$ of $f: X \rightarrow C$ as follows:

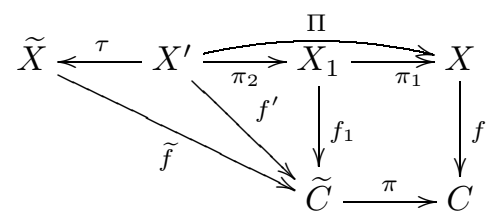

where $X_{1}=X \times_{C} \widetilde{C}, \pi_{1}$ and $f_{1}$ are the projections. $X^{\prime}$ is the minimal resolution of the singularities of the normalization of $X_{1}$ and $\tau$ is the contraction of those $(-1)$-curves in the fibers. Then we get the pullback fibration $\tilde{f}$ of $f$ under the base change $\pi$.

Now we consider the above construction locally. Let $F$ be a fiber of $f$ over $p \in C$. Assume that $\pi$ is totally ramified over $p$, i.e., $\pi^{-1}(p)$ contains only one point $\widetilde{p}$. In this case, $\pi$ is defined locally by $z=w^{n}$ near $p=0$.

Now denote by $\widetilde{F}$ (resp. $F^{\prime}$ ) the fiber of $\widetilde{f}$ (resp. $f^{\prime}$ ) over $\widetilde{p} \in \widetilde{C}$. In fact, $F^{\prime}=\frac{1}{n} \Pi^{*}(F)$.

Definition 2.3. The fiber $\widetilde{F}$ of $\widetilde{f}$ over $\widetilde{p}$ is called the $n$-th root model of $F$.

Note that $F$ and any of its normal crossing model $\bar{F}$ have the same $n$-th root model $\widetilde{F}$ for any $n$. In fact, if $F$ is normal crossing, then $F^{\prime}$ is also normal crossing. In particular, $\bar{F}^{\prime}$ is the normal crossing model of $\widetilde{F}$.

Indeed, we can assume that $F=\bar{F}=\sum_{i=1}^{k} n_{i} C_{i}$ is normal crossing, where $C_{i}$ is irreducible. Let $p$ be a singular point of $F_{\text {red }}$. Without loss of generality, we assume that $p$ is an intersection point of $C_{i}$ with $C_{j}$. Near $p, \pi_{1}$ is defined locally by $z^{n}=x^{n_{i}} y^{n_{j}}$. Then we see that the singularities of the normalization of $X_{1}$ are of Hirzebruch-Jung type. Hence, $F^{\prime}$ is normal crossing. By the computation of the normalization, we see that the multiplicity of the strict transform of $C_{i}$ in $F^{\prime}$ is $n_{i} / \operatorname{gcd}\left(n, n_{i}\right)$.

If $n_{i}$ divides $n$ for any $i$, then one can prove that $F^{\prime}$ and $\widetilde{F}$ are semistable. This is the famous Semistable Reduction Theorem. Denote by $M_{F}=\operatorname{lcm}\left\{n_{1}, \cdots, n_{k}\right\}$. Then the $n$-th root model of $F$ is always semistable if $n$ satisfies $n \equiv 0\left(\bmod M_{F}\right)$.

Definition 2.4. If $\widetilde{F}$ is semistable, then $\widetilde{F}$ is called the semistable model of $F$ or the semistable reduction of $F$.

\subsection{Dual model $F^{*}$ of $F$.}

Definition 2.5. If $n \equiv-1\left(\bmod M_{F}\right)$, then the $n$-th root model of $F$ is called the dual model of $F$, denoted by $F^{*}$.

The dual model is first introduced by Kodaira for elliptic fibrations. Our definition is a natural generalization. In general, $\left(F^{*}\right)^{*}$ doesn't coincide with $F$ unless the semistable model of $F$ is smooth. (If the uniqueness of the dual model is needed, one may choose $n$ to be the minimal positive integer satisfying $n \equiv-1\left(\bmod M_{F}\right)$.)

Let $\bar{F}=\sum_{i=1}^{k} n_{i} C_{i}$ be the minimal normal crossing model of $F$, where the $C_{i}$ 's are all irreducible components. We have seen that $\bar{F}^{\prime}$ is the normal crossing model of $F^{*}$. 
Let $n \equiv-1\left(\bmod M_{F}\right)$. Denote by $C_{i}^{*}$ the strict transform of $C_{i}$ in $\bar{F}^{\prime}$. Because $n_{i}$ is prime to $n$ for any $i, C_{i}^{*}$ is irreducible. The multiplicity of $C_{i}^{*}$ in $\bar{F}^{\prime}$ is still $n_{i}$. By the resolution of Hirzebruch-Jung singularities, we see that $\bar{F}^{\prime}$ is obtained by inserting a chain of rational curves,

$$
\bar{F}^{\prime}=\sum_{i=1}^{k} n_{i} C_{i}^{*}+\sum_{p} \Gamma_{p}^{*}
$$

where $p$ runs over all double points of $\bar{F}, \Gamma_{p}^{*}=\sum_{i=1}^{r} \gamma_{i} \Gamma_{i}$. Assume that $p$ is an intersection point of two local components $C_{i}$ and $C_{j}$. Then near $\Gamma_{p}^{*}, \bar{F}^{\prime}$ is as follows, where $\gamma_{0}=n_{i}$ and $\gamma_{r+1}=n_{j}$ :

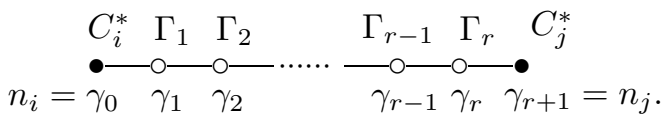

Lemma 2.6. 1) For $i=1, \cdots, r$, we have $\gamma_{i} \mid \gamma_{i-1}+\gamma_{i+1}$.

2) $\gamma_{0} \mid \gamma_{1}+\gamma_{r+1}$ and $\gamma_{r+1} \mid \gamma_{r}+\gamma_{0}$.

Proof. The local base change over $p$ is defined by $z^{n}=x^{n_{i}} y^{n_{j}}$. Note that $n$ is prime to $n_{i}$ and $n_{j}$, the equation is equivalent to $z^{n}=x y^{n-q}$ for some $q$ satisfying $n_{j}+q n_{i} \equiv 0(\bmod n)$ and $1 \leq q<n$ (see [5], Ch. III, §5). By definition, $n_{i}$ divides $n+1$. One can see that $q_{0}=-(n+1) n_{j} / n_{i}=-(n+1) \gamma_{r+1} / \gamma_{0}$ is an integer satisfying $q \equiv q_{0}(\bmod n)$. The singular point over $p$ is of Hirzebruch-Jung type.

For convenience, we take $\Gamma_{0}=C_{i}^{*}, \gamma_{0}=n_{i}, \Gamma_{r+1}=C_{j}^{*}$ and $\gamma_{r+1}=n_{j}$. Let $e_{i}=-\Gamma_{i}^{2}$. By Zariski's lemma ([5], Ch. III, §8), $\bar{F}^{\prime} \cdot \Gamma_{i}=0$ for $i=1, \cdots, r$. Thus we have

$$
\left\{\begin{array}{c}
-\gamma_{0}+\gamma_{1} e_{1}-\gamma_{2}=0 \\
-\gamma_{1}+\gamma_{2} e_{2}-\gamma_{3}=0 \\
\vdots \\
-\gamma_{r-1}+\gamma_{r} e_{r}-\gamma_{r+1}=0
\end{array}\right.
$$

So we have proved 1). For fixed $\gamma_{0}$ and $\gamma_{r+1}$, this is a linear system of the $r$ variables $\gamma_{1}, \cdots, \gamma_{r}$. We denote by $A=\left[e_{1}, \cdots, e_{r}\right]$ the coefficient matrix. It is well known that the determinant of $A$ is equal to $n$, and the determinant of the submatrix $\left[e_{2}, \cdots, e_{r}\right]$ is equal to $q$. By the Gramer Rule,

$$
\gamma_{1}=\frac{\gamma_{0} q+\gamma_{r+1}}{n}=\frac{\gamma_{0} q_{0}+\gamma_{r+1}}{n}+\gamma_{0} \frac{q-q_{0}}{n}=-\gamma_{r+1}+\gamma_{0} \frac{q-q_{0}}{n},
$$

so $\gamma_{0} \mid \gamma_{1}+\gamma_{r+1}$. Symmetrically, $\gamma_{r+1} \mid \gamma_{r}+\gamma_{0}$.

Lemma 2.7. The reduced normal crossing models of $F$ and $F^{*}$ have the same arithmetic genus, i.e., $p_{a}\left(\bar{F}_{\text {red }}\right)=p_{a}\left(\bar{F}^{\prime}\right.$ red $)$.

Proof. This follows from the fact that the arithmetic genus of $\bar{F}$ is equal to the sum of the geometric genus of each component plus the number of cycles in the dual graph of $\bar{F}$. Note that the geometric genera of $C_{i}$ and $C_{i}^{*}$ are the same. So the arithmetic genus is not changed by inserting a Hirzebruch-Jung chain of rational curves. 


\section{LOCAL INVARIANTS OF A FIBER}

In order to obtain the computation formulas for the Chern numbers of a singular fiber, we need to introduce several local invariants for a singular point of a curve, not necessarily reduced. See [13.

3.1. Invariants $\alpha$ and $\beta$ for a curve singularity. In Definition 2.1, we denote by $m_{i+1}$ the multiplicity of $\left(B_{i, \text { red }}, p_{i}\right)$ at $p_{i}$. (Note that $B_{i, \text { red }}$ is the reduced total transform of $B_{\text {red }}$, instead of the strict transform.) One can check that if $B$ is a compact curve, then

$$
p_{a}\left(B_{r, \text { red }}\right)=p_{a}\left(B_{\text {red }}\right)-\frac{1}{2} \sum_{i=1}^{r}\left(m_{i}-1\right)\left(m_{i}-2\right) .
$$

Suppose $B$ has only one singular point, $p=p_{0}$. Let $k_{p}=k_{p}(B)$ (resp. $\mu_{p}=$ $\left.\mu_{p}(B)\right)$ be the number of local branches (resp. Milnor number) of $\left(B_{\text {red }}, p\right)$. Then

$$
\mu_{p}=\sum_{i=1}^{r}\left(m_{i}-1\right)\left(m_{i}-2\right)+k_{p}-1 .
$$

1) $m_{i}=2$ for all $i$ if and only if $\left(B_{\text {red }}, p\right)$ is a node.

2) $m_{i} \leq 3$ for all $i$ if and only if $\left(B_{\text {red }}, p\right)$ is an $A D E$ singular point (5], Ch.II, $\S 8)$.

If $q \in B_{r, \text { red }}$ is a double point, and the two local components of $\left(B_{r}, q\right)$ have multiplicities $a_{q}$ and $b_{q}$, then we define $\left[a_{q}, b_{q}\right]:=\frac{\operatorname{gcd}\left(a_{q}, b_{q}\right)^{2}}{a_{q} b_{q}}$, and

$$
\alpha_{p}=\sum_{i=1}^{r}\left(m_{i}-2\right)^{2}, \quad \beta_{p}=\sum_{q \in B_{r}}\left[a_{q}, b_{q}\right],
$$

where $q$ runs over all of the double points of $B_{r, \text { red }}$. These two invariants are independent of the resolution.

In [13, we prove that $\mu_{p} \geq \alpha_{p}+\beta_{p}$. Actually, we need more precise inequalities of this kind.

Example 3.1. The invariants of an $A D E$ singularity $\left(B_{\text {red }}, p\right)$ are as follows:

\begin{tabular}{|c|c|c|c|c|c|c|c|}
\hline & $A_{2 k-1}$ & $A_{2 k}$ & $D_{2 k+2}$ & $D_{2 k+3}$ & $E_{6}$ & $E_{7}$ & $E_{8}$ \\
\hline$\mu_{p}$ & $2 k-1$ & $2 k$ & $2 k+2$ & $2 k+3$ & 6 & 7 & 8 \\
\hline$\alpha_{p}$ & $k-1$ & $k$ & $k$ & $k+1$ & 3 & 3 & 4 \\
\hline$\beta_{p}$ & $\mathrm{I}_{k}$ & $\frac{3 k}{2 k+1}$ & $\mathrm{II}_{k}$ & $\mathrm{III}_{k}$ & 1 & $\mathrm{IV}$ & $\frac{4}{5}$ \\
\hline$\beta_{p}^{-}$ & $\geq 1-\frac{1}{k}$ & $\geq \frac{6 k-1}{4 k+2}$ & & $\geq \frac{1}{2}$ & $\geq \frac{11}{12}$ & $\geq \frac{1}{3}$ & $\geq \frac{11}{15}$ \\
\hline
\end{tabular}

$$
\left\{\begin{array}{l}
\mathrm{I}_{k}=1-\frac{1}{k}+[k(n+m), n]+[k(n+m), m], \\
\mathrm{II}_{k}=\frac{k(n, m+l)^{2}}{n(n+k(m+l))}+[n+k(m+l), m]+[n+k(m+l), l], \\
\mathrm{III}_{k}=\frac{1}{2}+[m, 2((2 k+1) m+n)]+\frac{(2 k+1)(n, 2 m)^{2}}{2 n((2 k+1) m+n)}, \\
\mathrm{IV}=\frac{1}{3}+\frac{2(3 m, n)^{2}}{3 n(2 m+n)}+\frac{(m, 3 n)^{2}}{3 m(2 m+n)} .
\end{array}\right.
$$


Where $n$ (resp. $m$ or $l$ ) is the multiplicity of a local branch of $(F, p) . n$ corresponds to a smooth branch. We have

$$
\mathrm{I}_{k} \leq 1, \quad \mathrm{II}_{k} \leq 1, \quad \mathrm{III}_{k} \leq \frac{3(k+1)}{2 k+3}, \quad \mathrm{IV} \leq \frac{4}{5} .
$$

Lemma 3.2. 1) $\mu_{p} \geq \alpha_{p}+\beta_{p}$, with equality iff the singularity is of types $A_{1}$ or $A_{2}$.

2) $\mu_{p} \geq \alpha_{p}+\beta_{p}+1$ except for the singularities of types $A_{k}$ for $k \leq 4$.

3) $\mu_{p} \geq \alpha_{p}+\beta_{p}+2$ except for the singularities of types $A_{k}(k \leq 6)$ and $D_{5}$.

4) If $2\left(\mu_{p}-\alpha_{p}-\beta_{p}\right)+\alpha_{p}+3 \beta_{p}^{-}<6$, then $p$ is of types $A_{1}, A_{2}, A_{3}$ and $D_{4}$. If $2\left(\mu_{p}-\alpha_{p}-\beta_{p}\right)+\alpha_{p}+3 \beta_{p}^{-}<5$, then $p$ is of types $A_{1}, A_{2}$ and $A_{3}$. If $2\left(\mu_{p}-\alpha_{p}-\beta_{p}\right)+\alpha_{p}+3 \beta_{p}^{-}<\frac{7}{2}$, then $p$ is a node.

Proof. For an $A D E$ singular point $p$, the inequalities can be checked directly from the computation above.

If $p$ is not an $A D E$ singular point, then at least one $m_{i} \geq 4$, so $\alpha_{p} \geq 4$. We claim that $\mu_{p} \geq \alpha_{p}+\beta_{p}+2$. In Definition 2.1, we assume that $\sigma=\sigma_{1} \circ \sigma_{2}$, where $\sigma_{1}: X^{\prime} \rightarrow X$ consists of blowing-ups at the non- $A D E$ singular points $p_{0}, \cdots, p_{r^{\prime}-1}$ such that $B^{\prime}=\sigma_{1}^{*} B$ admits at worst $A D E$ singular points. Then we have

$$
\mu_{p}-\alpha_{p}-\beta_{p}=\sum_{i=1}^{r^{\prime}}\left(m_{i}-3\right)+\sum_{p^{\prime} \in B^{\prime}}\left(\mu_{p^{\prime}}-\alpha_{p^{\prime}}-\beta_{p^{\prime}}\right) .
$$

Because $p$ is not an $A D E$ singular point, at least one of $m_{i}\left(i \leq r^{\prime}\right)$ is larger than 3. If two of these $m_{i}$ 's are larger than 3 , then $\mu_{p} \geq \alpha_{p}+\beta_{p}+2$. Without loss of generality, we assume that $m_{1}=4$ and $r^{\prime}=1$. Namely $m_{1}=4$ and $m_{i} \leq 3$ for all $i \geq 2$. We can also assume that $\mu_{p^{\prime}}<\alpha_{p^{\prime}}+\beta_{p^{\prime}}+1$ for any singular point $p^{\prime}$ of $B_{\text {red }}^{\prime}$.

Now we consider the $A D E$ singular points of $B^{\prime}$. Because the exceptional curve is one of the branches of the singular points $p^{\prime}$ of $B_{\text {red }}^{\prime}$, each singular point $p^{\prime}$ has at least two branches. According to 1 ), the singular points $p^{\prime}$ of $B_{\text {red }}^{\prime}$ are of types $A_{1}$ or $A_{3}$. Note that if $p^{\prime}$ is of type $A_{3}$, then $\mu_{p^{\prime}}-\alpha_{p^{\prime}}-\beta_{p^{\prime}}=\frac{1}{2}$. Thus if $B^{\prime}$ admits at least two $A_{3}$, then $\mu_{p} \geq \alpha_{p}+\beta_{p}+2$ holds true.

If $B^{\prime}$ admits only one $A_{3}$, then we can assume that $(B, p)$ is defined by $(x-$ $y)^{a}(x+y)^{b}\left(x^{2}-y^{3}\right)^{c}=0$. Now it is easy to check that $\mu_{p}=10, \alpha_{p}=5$ and $\beta_{p} \leq 2$. So $\mu_{p} \geq \alpha_{p}+\beta_{p}+2$.

If $B^{\prime}$ admits no $A_{3}$, then $B^{\prime}$ admits $4 A_{1}$. Hence we can assume that $(B, p)$ is defined by $x^{a} y^{b}(x-y)^{c}(x+y)^{d}=0$. We have $\mu_{p}=9, \alpha_{p}=4$ and $\beta_{p} \leq 1$. Thus $\mu_{p} \geq \alpha_{p}+\beta_{p}+2$.

Lemma 3.3. A curve singularity $p$ satisfying $\sum_{i=1}^{r} m_{i}\left(m_{i}-2\right) \leq 5$ must be of types $A_{1}, A_{2}, A_{3}$ and $D_{4}$.

Proof. The condition implies that $m_{i} \leq 3$ for any $i$ and there exists at most one $i$ such that $m_{i}=3$, so $p$ is an $A D E$ singular point. Now one can check the result directly.

We define $\beta_{F}$ as the sum of $\beta_{p}$. One can easily check that $\beta_{F}$ is independent of the resolution; thus $F, \sigma^{*} F$ and $\bar{F}$ have the same $\beta$-invariant. 


\subsection{Invariants $\beta^{-}$and $\beta^{+}$.}

Definition 3.4. Let $\bar{F}$ be the minimal normal crossing model of $F$, and let $G(\bar{F})$ be the dual graph of $\bar{F}$. An H-J branch of rational curves in $G(\bar{F})$ is

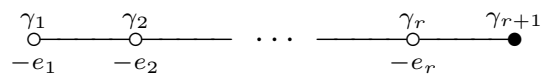

where $\underset{-e_{i}}{\stackrel{\gamma_{i}}{\stackrel{0}{0}}}$ denotes a smooth rational curve $\Gamma_{i}$ with $\Gamma_{i}^{2}=-e_{i}$ whose multiplicity in $\bar{F}$ is $\gamma_{i}$. • denotes either a curve $\Gamma \neq \mathbb{P}^{1}$ or a smooth rational curve meeting at 3 or more points with the other components. We call $\Gamma_{1}$ an end point of $G(\bar{F})$.

Note that the $r$ rational curves can be contracted to a Hirzebruch-Jung singularity of type $(n, q)$ with defining equation $z^{n}=x y^{n-q}([5$, Ch. III, $\S 5)$, where $n$ and $q$ are respectively the determinants of the matrices $\left[e_{1}, \cdots, e_{r}\right]$ and $\left[e_{2}, \cdots, e_{r}\right] . n$ and $q$ can also be determined by the multiplicities $\gamma_{i}$ as follows.

According to (2.1) and $\gamma_{0}=0$, we see that $\gamma_{1}$ divides $\gamma_{i}$ for any $i$. Using the notation of ([5], Ch. III, §5), $\gamma_{1}<\gamma_{2}<\cdots<\gamma_{r}, \gamma_{i}=\mu_{i} \gamma_{1}$ for any $i$, so $1=\mu_{1}<\mu_{2}<\cdots<\mu_{r+1}$.

$$
n=\mu_{r+1}=\frac{\gamma_{r+1}}{\gamma_{1}}, \quad q^{\prime}=\mu_{r}=\frac{\gamma_{r}}{\gamma_{1}},
$$

and $q$ is the unique solution of the equation

$$
q q^{\prime} \equiv 1 \quad(\bmod n), \quad 1 \leq q<n .
$$

Since $\mu_{i}$ and $\mu_{i+1}$ are coprime, the contribution of the branch to $\beta_{F}=\beta_{\bar{F}}$ is

$$
\beta^{\prime}=\frac{1}{\mu_{1} \mu_{2}}+\frac{1}{\mu_{2} \mu_{3}}+\cdots+\frac{1}{\mu_{r} \mu_{r+1}} .
$$

There is a relation ([5], Ch. III, $\S 5$, eq. (6))

$$
\lambda_{k} \mu_{k+1}-\lambda_{k+1} \mu_{k}=n,
$$

i.e.,

$$
\frac{\lambda_{k}}{\mu_{k}}-\frac{\lambda_{k+1}}{\mu_{k+1}}=n \frac{1}{\mu_{k} \mu_{k+1}} .
$$

Note that $\lambda_{1}=q$ and $\lambda_{r+1}=0$. Take the sum of (3.7) from $k=1$ to $r$; we have

$$
\beta^{\prime}=\frac{1}{n}\left(\frac{\lambda_{1}}{\mu_{1}}-\frac{\lambda_{r+1}}{\mu_{r+1}}\right)=\frac{q}{n} .
$$

Lemma 3.5. The contribution of the $H-J$ branch to $\beta_{F}$ is $\frac{q}{n}$.

Definition 3.6. $\beta_{F}^{-}=\sum \beta^{\prime}$ is the total contribution of all H-J branches in $G(\bar{F})$. $\frac{1}{e_{1}}$.

Note that $\gamma_{2}=e_{1} \gamma_{1}$; the contribution of an H-J branch to $\beta_{F}^{-}$is at least $\left[\gamma_{1}, \gamma_{2}\right]=$

Example 3.7. If $e_{1}=\cdots=e_{r-1}=2$ and $e_{r} \geq 2$, then $n=r\left(e_{r}-1\right)+1$, $q=n-\left(e_{r}-1\right)=(r-1)\left(e_{r}-1\right)+1$, and the contribution of this H-J branch to $\beta_{F}^{-}$is

$$
\beta^{\prime}=\frac{(r-1)\left(e_{r}-1\right)+1}{r\left(e_{r}-1\right)+1}=1-\frac{e_{r}-1}{r\left(e_{r}-1\right)+1} .
$$


Theorem 3.8 (Gang Xiao [18). Assume that $n \equiv 0\left(\bmod M_{F}\right)$. Let $\bar{F}$ be the minimal normal crossing model of $F$. Consider the construction of the $n$-th root model of $\bar{F}$ as in $\S 2.2$. Then a curve in $X^{\prime}$ is contracted by $\tau$ if and only if it comes from an $H-J$ branch in $\bar{F}$.

The theorem above is contained in the proof of Prop. 1 of [18].

From the previous theorem, $\beta_{F}^{-}$is just $c_{-1}(F)$ defined in [13] by the remark of $\left([13]\right.$, p. 666), i.e., $n \beta_{F}^{-}$is the number of $(-1)$-curves contracted by $\tau$. Let $\beta_{F}^{+}=\beta_{F}-\beta_{F}^{-}$. Then

$$
\beta_{F}=\beta_{F}^{+}+\beta_{F}^{-} .
$$

3.3. Formulas for the Chern numbers of a fiber. Let $\mu_{F}=\sum_{p} \mu_{p}\left(F_{\text {red }}\right)$ be the sum of the Milnor numbers of the singularities of $F_{\text {red }}$.

Let $N_{F}=g-p_{a}\left(F_{\text {red }}\right)$. One can prove that $0 \leq N_{F} \leq g . N_{F}=0$ iff $F$ is reduced or $g=1$ and $F$ is of type ${ }_{m} \mathrm{I}_{b} . N_{F}=g$ iff $F$ is a tree of smooth rational curves.

The topological characteristic of $F$ is equal to $2 N_{F}+\mu_{F}+2-2 g$.

Then we have the following formulas for the computation of the Chern numbers of $F$ :

$$
\left\{\begin{array}{l}
c_{1}^{2}(F)=4 N_{F}+F_{\text {red }}^{2}+\alpha_{F}-\beta_{F}^{-}, \\
c_{2}(F)=2 N_{F}+\mu_{F}-\beta_{F}^{+}, \\
12 \chi_{F}=6 N_{F}+F_{\text {red }}^{2}+\alpha_{F}+\mu_{F}-\beta_{F} .
\end{array}\right.
$$

From the blow-up formulas, we only need to compute the Chern numbers of the minimal normal crossing model $\bar{F}$.

\section{Proof of Theorem 1.1}

4.1. Dedekind's reciprocity law. We denote by $(p, q)$ the greatest common divisor of two integers $p$ and $q$. The following notation is from Dedekind's Reciprocity Law. Take

$$
\chi(p, q)=\frac{1}{12}\left(\frac{q}{p}+\frac{p}{q}+\frac{(p, q)^{2}}{p q}\right)-\frac{1}{4} .
$$

One can easily check the following identities:

$$
\chi(p, p)=0, \quad \chi(p, q)=\chi(p, p+q)+\chi(p+q, q) .
$$

If $p$ and $q$ are coprime, then Dedekind's sum is defined as follows:

$$
s(p, q)=\sum_{i=0}^{q-1}\left(\left(\frac{p i}{q}\right)\right)\left(\left(\frac{i}{q}\right)\right),
$$

where

$$
((x))= \begin{cases}x-[x]-\frac{1}{2}, & x \notin \mathbb{Z}, \\ 0, & x \in \mathbb{Z},\end{cases}
$$

and $[x]$ is the largest integer $\leq x .((x))$ is an odd function since $((-x))=-((x))$ and is periodic with period 1 .

If $p$ and $q$ are not coprime, then we define $s(p, q):=s(p /(p, q), q /(p, q))$. Therefore, $s(-p, q)+s(p, q)=0$, and $s(p+k q, q)=s(p, q)$ for all integers $k$. In particular, if $p+p^{\prime}$ is divisible by $q$, then

$$
s(p, q)+s\left(p^{\prime}, q\right)=0 .
$$


The well-known Dedekind's Reciprocity Law says

$$
s(p, q)+s(q, p)=\chi(p, q) .
$$

4.2. Compute $\chi_{F}$ from the normal crossing model $\bar{F}$. Let $F$ be a singular fiber and $\bar{F}=\sum_{i=1}^{k} n_{i} C_{i}$ be the normal crossing model of $F$, where the $C_{i}$ 's are all irreducible components. Take $M_{F}=\operatorname{lcm}\left(n_{1}, \cdots, n_{k}\right)$.

Theorem 4.1. Let $N_{\bar{F}}=g-p_{a}\left(\bar{F}_{\text {red }}\right)$. Then

$$
\chi_{F}=\frac{1}{2} N_{\bar{F}}-\sum_{i<j} \chi\left(n_{i}, n_{j}\right) C_{i} C_{j} .
$$

Proof. Note that $\chi_{F}$ is a birational invariant, so

$$
\chi_{F}=\chi_{\bar{F}}=\frac{1}{2} N_{\bar{F}}+\frac{1}{12}\left(\mu_{\bar{F}}-\beta_{\bar{F}}+\bar{F}_{\text {red }}^{2}\right) .
$$

By definition,

$$
\mu_{\bar{F}}=\sum_{i<j} C_{i} C_{j}, \quad \beta_{\bar{F}}=\sum_{i<j} \frac{\left(n_{i}, n_{j}\right)^{2}}{n_{i} n_{j}} C_{i} C_{j}, \quad \bar{F}_{\text {red }}^{2}=\sum_{i<j} 2 C_{i} C_{j}+\sum_{i=1}^{k} C_{i}^{2} .
$$

Since $C_{i} \bar{F}=0, C_{i}^{2}=-\sum_{j \neq i} \frac{n_{j}}{n_{i}} C_{i} C_{j}$, we have $\sum_{i=1}^{k} C_{i}^{2}=-\sum_{i<j}\left(\frac{n_{i}}{n_{j}}+\frac{n_{j}}{n_{i}}\right) C_{i} C_{j}$. Thus

$$
\mu_{\bar{F}}-\beta_{\bar{F}}+\bar{F}_{\text {red }}^{2}=\sum_{i<j}\left(3-\frac{\left(n_{i}, n_{j}\right)^{2}}{n_{i} n_{j}}-\frac{n_{j}}{n_{i}}-\frac{n_{i}}{n_{j}}\right) C_{i} C_{j}=-12 \sum_{i<j} \chi\left(n_{i}, n_{j}\right) C_{i} C_{j} .
$$

Hence $\chi_{F}=\frac{1}{2} N_{\bar{F}}-\sum_{i<j} \chi\left(n_{i}, n_{j}\right) C_{i} C_{j}$.

\subsection{Duality theorem for $\chi$.}

Theorem 4.2. $F^{*}$ is the dual fiber of $F$. Then $\chi_{F}+\chi_{F^{*}}=N_{\bar{F}}=N_{\bar{F}^{*}}$.

Proof. We use the notation in 2.3 . We have seen that the normal crossing model $\bar{F}^{*}$ of $F^{*}$ is of the following type:

$$
\bar{F}^{*}=\sum_{i=1}^{k} n_{i} C_{i}^{*}+\sum_{p} \Gamma_{p}^{*}
$$

where $p$ runs over all double points of $\bar{F}$, and $\Gamma_{p}^{*}=\gamma_{1} \Gamma_{1}+\cdots+\gamma_{r} \Gamma_{r}$ is as follows:

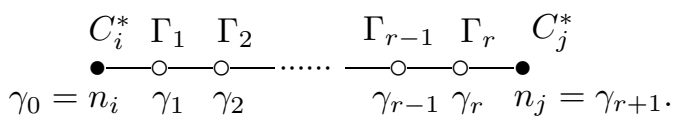

By 1) of Lemma 2.6. where if $i=1, \cdots, r$, then $\gamma_{i}$ divides $\gamma_{i-1}+\gamma_{i+1}$, we have

$$
s\left(\gamma_{i-1}, \gamma_{i}\right)+s\left(\gamma_{i+1}, \gamma_{i}\right)=0, \quad \text { for } i=1, \cdots, r .
$$

By 2) of Lemma 2.6, we have

$$
s\left(\gamma_{1}, \gamma_{0}\right)=-s\left(\gamma_{r+1}, \gamma_{0}\right), \quad s\left(\gamma_{r}, \gamma_{r+1}\right)=-s\left(\gamma_{0}, \gamma_{r+1}\right) .
$$


Hence

$$
\begin{aligned}
\sum_{i=1}^{r+1} \chi\left(\gamma_{i-1}, \gamma_{i}\right) \Gamma_{i-1} \Gamma_{i} & =\sum_{i=1}^{r+1}\left(s\left(\gamma_{i-1}, \gamma_{i}\right)+s\left(\gamma_{i}, \gamma_{i-1}\right)\right) \\
& =s\left(\gamma_{1}, \gamma_{0}\right)+s\left(\gamma_{r}, \gamma_{r+1}\right)+\sum_{i=1}^{r}\left(s\left(\gamma_{i-1}, \gamma_{i}\right)+s\left(\gamma_{i+1}, \gamma_{i}\right)\right) \\
& =-s\left(\gamma_{r+1}, \gamma_{0}\right)-s\left(\gamma_{0}, \gamma_{r+1}\right)=-\chi\left(n_{i}, n_{j}\right) .
\end{aligned}
$$

Thus

$$
\mu_{\bar{F}^{*}}-\beta_{\bar{F}^{*}}+\bar{F}^{*}{ }_{\text {red }}^{2}=-\left(\mu_{\bar{F}}-\beta_{\bar{F}}+\bar{F}_{\text {red }}^{2}\right) .
$$

By Lemma 2.7. $p_{a}\left(\bar{F}^{*}\right.$ red $)=p_{a}\left(\bar{F}_{\text {red }}\right)$, so $N_{\bar{F}^{*}}=N_{\bar{F}}$. We get $\chi_{F}+\chi_{F^{*}}=N_{\bar{F}}$.

\subsection{Upper and lower bounds on $\chi$.}

Theorem 4.3. $\frac{1}{6} N_{\bar{F}} \leq \chi_{F} \leq \frac{5}{6} N_{\bar{F}}$. If $F$ is not semistable, then $\frac{1}{6} \leq \chi_{F} \leq \frac{5 g}{6}$.

Proof. By the adjunction formula, $2 N_{F}=K_{X}\left(F-F_{\text {red }}\right)-F_{\text {red }}^{2}$. By the resolution of the singularities of $F$, we have $p_{a}\left(F_{\text {red }}\right)=p_{a}\left(\bar{F}_{\text {red }}\right)-\sum_{i} \frac{1}{2}\left(m_{i}-1\right)\left(m_{i}-2\right)$, so $2 N_{F}=2 N_{\bar{F}}-\sum_{i}\left(m_{i}-1\right)\left(m_{i}-2\right)$, where $m_{i} \geq 2$ are the multiplicities of singularities occurring in the partial resolutions of $F$. By definition, $\alpha_{F}=\sum_{i}\left(m_{i}-2\right)^{2}$. From formulas (1.3),

$$
\begin{aligned}
12 \chi_{F} & =6 N_{F}+F_{\text {red }}^{2}+\alpha_{F}+\mu_{F}-\beta_{F} \\
& =2 N_{F}+\left(2 N_{F}+F_{\text {red }}^{2}\right)+\left(\mu_{F}-\alpha_{F}-\beta_{F}\right)+\left(2 N_{F}+\alpha_{F}\right) \\
& =2 N_{F}+\left(F-F_{\text {red }}\right) K_{X}+\left(\mu_{F}-\alpha_{F}-\beta_{F}\right)+2 N_{\bar{F}}+\sum_{i}\left(m_{i}-2\right)\left(m_{i}-3\right) .
\end{aligned}
$$

Since $F$ is minimal, $\left(F-F_{\text {red }}\right) K_{X} \geq 0 . \mu_{F}-\alpha_{F}-\beta_{F} \geq 0$ is proved in Lemma 3.2 . Hence $12 \chi_{F} \geq 2 N_{\bar{F}}$.

Similarly, $12 \chi_{F^{*}} \geq 2 N_{\bar{F}^{*}}=2 N_{\bar{F}}$. On the other hand, $\chi_{F}+\chi_{F^{*}}=N_{\bar{F}}$, so $12 \chi_{F} \leq 10 N_{\bar{F}}$.

Corollary 4.4. $\chi_{F}=\frac{1}{6} N_{\bar{F}}$ (resp. $\left.\chi_{F}=\frac{5}{6} N_{\bar{F}}\right)$ if and only if $F$ (resp. $F^{*}$ ) is a reduced curve whose singularities are at worst ordinary cusps or nodes.

Proof. It follows from Lemma 3.2 .

\subsection{Applications.}

Theorem 4.5. 1) If $f$ is non-trivial, then $\chi_{f} \leq \frac{g}{2}\left(2 b-2+\frac{8}{3} s\right)$.

2) If $f$ is isotrivial, then $\chi_{f} \leq \frac{5 g s}{6}$.

Proof. 1) We first assume that $f$ is non-isotrivial. Let $F_{1}, \cdots, F_{s}$ be all of the singular fibers. There exists some semistable reduction $\pi: \tilde{C} \rightarrow C$ such that

(i) $\pi$ is ramified uniformly over the $s$ critical points of $f$, and the ramification index of $\pi$ at any ramified point is exactly $e$.

(ii) $e$ is divisible by $M_{F_{i}}$ for all $i$, and it can be arbitrarily large.

In fact, if $b=g(C)>0$, the existence follows from Kodaira-Parshin's construction; if $b=0$, then $s \geq 3$. Thus one can construct a base change totally ramified over the $s$ points. The existence is induced to the case $b>0$. 
Let $\tilde{f}: \tilde{S} \rightarrow \tilde{C}$ be the semistable model and $\tilde{s}$ be the number of singular fibers of $\tilde{f}$. Let $\tilde{b}=g(\tilde{C})$ and $d=\operatorname{deg} \pi$. One has

$$
2 \tilde{b}-2=d(2 b-2)+d\left(1-\frac{1}{e}\right) s, \quad \tilde{s} \leq \frac{d s}{e} .
$$

Hence we have

$\chi_{f}-\frac{g}{2}\left(2 b-2+\frac{8}{3} s\right)=\frac{1}{d}\left(\chi_{\tilde{f}}-\frac{g}{2}(2 \tilde{b}-2+\tilde{s})\right)+\frac{g}{2 d}\left(\tilde{s}-\frac{d s}{e}\right)+\sum_{i=1}^{s}\left(\chi_{F_{i}}-\frac{5 g}{6}\right)$.

$\chi_{\tilde{f}} \leq \frac{g}{2}(2 \tilde{b}-2+\tilde{s})$ is the Arakelov inequality, so one gets inequality (1).

2) It is obvious. If $f$ is also non-trivial, then 3) of Theorem 1.2 implies 2).

\section{Proof of Theorem 1.3}

5.1. Fibers with high $c_{1}^{2}$. We try to prove Theorem 1.3. which implies Theorem 1.2 , 2). To describe a fiber $F$, we usually consider the dual graph of its normal crossing model $\bar{F}$. We use $\circ$ to denote a $(-2)$-curve, and $\bullet$ a smooth rational curve but not a $(-2)$-curve. The number beside is the multiplicity of the curve in $\bar{F}$. The self-intersection number of each component • can be determined by using Zariski's lemma.

The following fiber $F$ of genus $g$ satisfies $c_{1}^{2}(F)=4 g-\frac{11}{2}, c_{2}(F)=2 g+\frac{5}{2}$, $\chi_{F}=\frac{g}{2}-\frac{1}{4}$.

Example 5.1. $F=(g-1) F_{0}$, where $F_{0}$ is a curve of genus 2 whose dual graph is as follows:

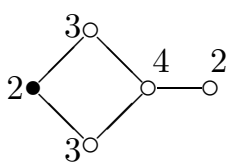

Lemma 5.2 (Artin [1]). Let $D$ be an effective divisor on a surface. Suppose $D^{2}<0$ and $D \Gamma_{i} \leq 0$ for any component $\Gamma_{i}$ of $D$. Then $D$ is a negative curve, i.e., the intersection matrix $\left(\Gamma_{i} \Gamma_{j}\right)$ is negative definite.

In what follows, we always assume that $F$ satisfies $c_{1}^{2}(F)>4 g-\frac{11}{2}$, namely,

$$
4 p_{a}\left(\bar{F}_{\text {red }}\right)-F_{\text {red }}^{2}+\beta_{F}^{-}+\sum_{i=1}^{r} m_{i}\left(m_{i}-2\right)<\frac{11}{2} .
$$

Note that each term on the left hand side of (5.1) is non-negative.

Lemma 5.3. 1) $m_{i} \leq 3$ for all $i$ and at most one $m_{i}$ is equal to 3 . So $F_{\text {red }}$ admits at most one singular point $p$ which is not a node. In fact, $p$ is of types $A_{2}, A_{3}$ or $D_{4}$.

2) $\bar{F}_{\text {red }}^{2} \leq-1$.

3) $p_{a}\left(\bar{F}_{\text {red }}\right)=0$, so $\bar{F}$ is a tree of smooth rational curves.

4) $p_{a}\left(F_{\text {red }}\right) \leq 1$, with equality iff one singular point $p$ of $F_{\text {red }}$ is not a node as in 1). 
Proof. 1) follows from the inequality $\sum_{i=1}^{r} m_{i}\left(m_{i}-2\right)<11 / 2$ and Lemma 3.3.

2) (5.1) implies that $p_{a}\left(\bar{F}_{\text {red }}\right) \leq 1$, i.e., $K \bar{F}_{\text {red }}+\bar{F}_{\text {red }}^{2} \leq 0$. If $\bar{F}_{\text {red }}^{2}=0$, then by Zariski's lemma, $\bar{F}=n \bar{F}_{\text {red }}$ for some positive integer $n$. Since $K \bar{F}_{\text {red }} \leq 0$, we see that $2 g-2=K \bar{F}=n K \bar{F}_{\text {red }} \leq 0$, a contradiction. So $\bar{F}_{\text {red }}^{2} \leq-1$.

3) Note that $p_{a}\left(\bar{F}_{\text {red }}\right) \leq 1$. Suppose that $p_{a}\left(\bar{F}_{\text {red }}\right)=1$. Then $\sum_{i=1}^{r} m_{i}\left(m_{i}-2\right) \leq$ $3 / 2$, so all $m_{i}=2$ and $F_{\text {red }}=\bar{F}_{\text {red }}$ is a nodal curve. We also see that $-F_{\text {red }}^{2}<3 / 2$, so $F_{\text {red }}^{2}=-1, K F_{\text {red }}=1$, and $F$ consists of one $(-3)$-curve and some $(-2)$-curves. Now from (5.1), we get $\beta_{F}^{-}<\frac{1}{2}$.

If one (-2)-curve $E$ in $F$ meets with the other components at only one point, then $E$ is the end point of some H-J branch, and the contribution of $E$ to $\beta_{F}^{-}$is at least $\frac{1}{2}$, a contradiction. Hence any $(-2)$-curve is a point in some loops in the dual graph of $F$. Because $p_{a}\left(F_{\text {red }}\right)=1$, there is only one loop in the dual graph. Hence the dual graph of $F$ consists of one loop. Now we see that $F_{\text {red }} \Gamma \leq 0$ for each irreducible component $\Gamma$. Combining with $F_{\text {red }}^{2}<0$, we know that $F$ is a negative curve (Lemma 5.2), a contradiction. 1.

4) By Lemma5.3 and (3.1), we have $p_{a}\left(F_{\text {red }}\right)=p_{a}\left(\bar{F}_{\text {red }}\right)+\sum_{i} \frac{1}{2}\left(m_{i}-1\right)\left(m_{i}-2\right) \leq$

\subsection{The case $p_{a}\left(F_{\text {red }}\right)=1$.}

Proposition 5.4. If $F$ is not a nodal curve, then $F_{\text {red }}$ has one singular point of type $A_{3}$. The normal crossing model of $F$ is of type 21.

Proof. In this case, $F$ has a unique singularity $p$ of types $A_{2}, A_{3}$, or $D_{4} \cdot p_{a}\left(F_{\text {red }}\right)=$ 1 ; one has $-F_{\text {red }}^{2}+\beta_{F}^{-}<\frac{5}{2}$. Since $p_{a}\left(\bar{F}_{\text {red }}\right)=0$, the dual graph of $\bar{F}$ is a tree of rational curves.

Case $A_{2}$. Suppose that $p$ is of type $A_{2}$. Then the contribution of $p$ to $\beta_{F}^{-} \geq \frac{5}{6}$, so $-F_{\text {red }}^{2}<\frac{5}{3}$. We have $-F_{\text {red }}^{2}=F_{\text {red }} K_{X}=1$, and $\beta_{F}^{-}<\frac{3}{2}$. Let $C_{1}$ be the irreducible component passing through $p$. Then $K_{X} C_{1}=1$ and $F_{\text {red }}-C_{1}$ is composed of some $A D E$ curves. Suppose that $F_{\text {red }}-C_{1}$ contains at least two $(-2)$-curves as the end points in the dual graph of $F$. Then their contributions to $\beta_{F}^{-}$is at least 1 . So $\beta_{F}^{-} \geq 1+\frac{5}{6}>\frac{3}{2}$, a contradiction. So only one $(-2)$-curve is an end point. On the other hand, from $p_{a}\left(\bar{F}_{\text {red }}\right)=0$, we see that $F$ contains no loop. Hence $F$ is an H-J chain with an end point $C_{1}$. It implies $F$ is a negative curve, a contradiction.

Case $A_{3}$. Assume that $p$ is of type $A_{3}$. The contribution of $p$ to $\beta_{F}^{-} \geq \frac{1}{2}$, and so $-F_{\text {red }}^{2}<2$. Now we have $-F_{\text {red }}^{2}=F_{\text {red }} K_{X}=1$ and $\beta_{F}^{-}<\frac{3}{2}$. $F$ consists of some $(-2)$-curves and one curve $C_{1}$ passing through $p$. Note that $\bar{F}$ is a tree of rational curves, so no node is a singular point of $C_{1}$, namely $C_{1}$ is smooth except at $p$. If $C_{1}$ is singular at $p$, then there is no $(-2)$-curve passing through $p$. Similar to the discussion above, only one $(-2)$-curve is the end point. Now we know that $F$ is a chain of $(-2)$-curves and $C_{1}$, so $F$ is a negative curve, a contradiction. Hence $C_{1}$ is smooth at $p$ and there is a (-2)-curve $C_{2}$ tangent to $C_{1}$ at $p$. Because $K_{X} C_{1}=1$, $C_{1}$ is a $(-3)$-curve.

There is a (-2)-curve $C_{2}$ tangent to $C_{1}$ at $p . F_{\text {red }}-C_{1}-C_{2}$ consists of $A D E$ curves. Because only one $(-2)$-curve is the end point, we know that $\Gamma=F_{\text {red }}-$ $C_{1}-C_{2}$ is just a curve of type $A_{n}$. 
If $C_{1}$ intersects $\Gamma$, then $F_{\text {red }}=\Gamma+C_{1}+C_{2}$ is a chain. One can prove that $F$ is a negative curve by Lemma 5.2 , a contradiction. So $C_{1}$ is disjoint with $\Gamma$. $C_{2}+\Gamma$ is a connected curve of type $A_{n+1}$.

By using Zariski's lemma, one can determine the multiplicities of all irreducible components in $F$ and the number of $(-2)$-curves. Finally, we get the fiber of type 21.

Case $D_{4}$. Suppose that $p$ is of type $D_{4}$. Because $\bar{F}$ is a tree of rational curves, the three local branches of $F$ at $p$ come from three different components, $C_{1}, C_{2}$ and $C_{3}$ of $F$. At least one component, say $C_{1}$, is not a (-2)-curve since $g \geq 2$. Suppose that $C_{2}$ is not a $(-2)$-curve. Then $F_{\text {red }} K_{X} \geq 2$. Recall that $F_{\text {red }} K_{X}=-F_{\text {red }}^{2} \leq 2$; one has $F_{\text {red }} K_{X}=-F_{\text {red }}^{2}=2$. Thus $\beta_{F}^{-}<\frac{1}{2}$ and $C_{1} K_{X}=C_{2} K_{X}=1$, namely, $C_{1}$ and $C_{2}$ are $(-3)$-curves. Hence $F_{\text {red }}-C_{1}-C_{2}$ consists of $A D E$-curves whose contributions to $\beta_{F}^{-} \geq \frac{1}{2}$, a contradiction. Therefore $C_{2}$ and $C_{3}$ must be $(-2)$ curves. Similarly, we can also prove that $C_{1}$ is not a $(-4)$-curve, hence it is a $(-3)$-curve. One can also prove that the other curves in $F$ are $(-2)$-curves. Now we have $-F_{\text {red }}^{2}=K F_{\text {red }}=1$, and $\beta_{F}^{-}<\frac{3}{2}$.

The normal crossing model $\bar{F}$ of $F$ is obtained by blowing up $F$ at $p$. Since the intersection matrix of $C_{1}, C_{2}$ and $C_{3}$ is negative definite, $\Gamma=F_{\text {red }}-C_{1}-C_{2}-C_{3}$ consists of $s \geq 1$ connected $A D E$-curves $\Gamma_{1}, \cdots, \Gamma_{s}$. From $\beta_{F}^{-}<\frac{3}{2}$, we see that at most two end points are $(-2)$-curves, so $s \leq 2$. Let $r_{i}-1$ be the number of irreducible components of $\Gamma_{i}$.

Suppose $s=2$. Since at most two end points are (-2)-curves, $\Gamma_{1}$ and $\Gamma_{2}$ are of types $A_{r_{1}-1}$ and $A_{r_{2}-1}$ respectively. In $\bar{F}, C_{1}^{2}=-4, C_{2}^{2}=C_{3}^{2}=-3$. $\Gamma_{i}$ meets $C_{j}$ at one point, so we obtain an H-J branch of type $\left[2,2, \cdots, 2, e_{r_{i}}\right]$, where $e_{r_{i}}=-C_{j}^{2}$.

Symmetrically, we only need to consider two cases: I) $\Gamma_{1}$ meets $C_{2}$ and $\Gamma_{2}$ meets $C_{1}$; II) $\Gamma_{1}$ meets $C_{2}$ and $\Gamma_{2}$ meets $C_{3}$.

In case I), from Zariski's lemma, one can find an equality $\frac{2}{3}=\frac{r_{1}}{2 r_{1}+1}+\frac{r_{2}}{3 r_{2}+1}$, i.e., $1=\frac{3}{2 r_{1}+1}+\frac{2}{3 r_{2}+1}$. We claim that there are no non-negative integers $r_{1}$ and $r_{2}$ satisfying this equation. Indeed, for $r_{1}=0,1$ or 2 , this equation has no nonnegative integral solution $r_{2}$. So we can assume that $r_{1} \geq 3$. Similarly, we can also assume that $r_{2} \geq 2$. Now the right hand side is less than 1 . So case I) does not occur.

In case II), we have similarly $\frac{3}{4}=\frac{r_{1}}{2 r_{1}+1}+\frac{r_{2}}{2 r_{2}+1}$, i.e., $\frac{1}{2}=\frac{1}{2 r_{1}+1}+\frac{1}{2 r_{2}+1}$. It is obvious that this equation has no integral solutions. So case II) cannot occur.

Suppose $s=1$. If $\Gamma_{1}$ is of type $A_{r_{1}-1}$, by Zariski's lemma, we have either $\frac{12}{5}=\frac{2 r_{1}+1}{r_{1}}$ or $3=3+\frac{1}{r_{1}}$. These equations have no integral solutions. So this case does not occur.

Finally, we assume that $\Gamma_{1}$ is not of type $A_{r_{1}-1}$. Now we see that there are two end points which are $(-2)$-curves, so the contribution of them to $\beta_{F}^{-}$is at least 1 . On the other hand, the contribution of the two components disjoint from $\Gamma_{1}$ are at least $\frac{1}{4}+\frac{1}{3}=\frac{7}{12}$. So $\beta_{F}^{-} \geq 1+\frac{7}{12}>\frac{3}{2}$, a contradiction.

Up to now, we have proved that the case $D_{4}$ does not occur.

5.3. The case $p_{a}\left(F_{\text {red }}\right)=0$. From now on, we always assume that $F_{\text {red }}$ is a tree of smooth rational curves, namely, $p_{a}\left(F_{\text {red }}\right)=0$. Hence (5.1) becomes $-F_{\text {red }}^{2}+\beta_{F}^{-}<$ $\frac{11}{2}$. Namely,

$$
F_{\text {red }} K_{X}+\beta_{F}^{-}<\frac{7}{2} .
$$


Lemma 5.5. We have $F_{\text {red }} K_{X}=1$ and $F_{\text {red }}^{2}=-3$. Namely, $F_{\text {red }}$ consists of a $(-3)$-curve and some $(-2)$-curves. So $\beta_{F}^{-}<\frac{5}{2}$.

Proof. Suppose that $F_{\text {red }} K_{X} \geq 2$. Let $s$ be the number of $(-2)$-curves as the end points in the dual graph of $F . \beta_{F}^{-}<\frac{3}{2}$ implies $s \leq 2$. Assume that the dual graph of $F$ contains $r$ end points. Obviously $r \geq 3$.

We first claim that $r=3, s=1$ and $F_{\text {red }} K_{X}=2$.

Indeed, there are at least $r-s$ end points which are not $(-2)$-curves. So $F_{\text {red }} K_{X} \geq r-s$ and $\beta_{F}^{-}<\frac{7}{2}+s-r$. On the other hand, $\beta_{F}^{-}>\frac{s}{2}$. So $s \geq 2 r-6$. Note that $s \leq 2$; we get $r \leq 4$. If $r=4$, then $s=2$. Then we see that $1<\beta_{F}^{-}<\frac{3}{2}$ and $F_{\text {red }} K_{X}=2$. It also implies that two of the end points are $(-3)$-curves. Thus $\beta_{F}^{-} \geq 2\left(\frac{1}{2}+\frac{1}{3}\right)>\frac{3}{2}$, a contradiction. So $r=3$.

If $F_{\text {red }} K_{X}=3$, then $\beta_{F}^{-}<\frac{1}{2}$. So any end point is a $(-3)$-curve. Thus $\beta_{F}^{-} \geq 1$, a contradiction. Hence $F_{\text {red }} K_{X}=2$. It implies $s \geq r-F_{\text {red }} K_{X}=1$.

Suppose that $s=2$. Since $r=3$ and $F$ is a tree of rational curves, $F$ has two H-J chains of type $A_{n}$ and one H-J chain whose end point is a $(-e)$-curve, $e=3$ or 4 . We have seen in $\$ 3.2$ that the multiplicities in an H-J branch increase strictly from the end point to the other side.

Suppose $e=4$. From $F_{\text {red }} K_{X}=2$, we see that all other components are (-2)curves. The dual graph of $F$ is as follows:

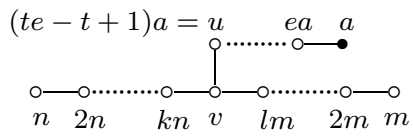

where $(k+1) n=(l+1) m=((t+1) e-t) a=v(1 \leq k \leq l)$ and $k n+l m+u=2 v$ by Zariski's lemma, so we have $\frac{k}{k+1}+\frac{l}{l+1}+\frac{u}{v}=2$. It is easy to see that

$$
\frac{k}{k+1}+\frac{l}{l+1}+\frac{1}{4} \leq \beta_{F}^{-}<\frac{3}{2} \text {. }
$$

So either $k=l=1$, or $k=1$ and $l=2$. Now we see that $\frac{u}{v}=1$ or $\frac{5}{6}$, a contradiction.

If $e=3$, then there exists another $(-3)$-curve $E$. In fact, $E$ cannot be in the center, otherwise $3 v=k n+l m+u<v+v+v$, a contradiction. $E$ cannot be in the vertical branch. Otherwise, we have

$$
\frac{k}{k+1}+\frac{l}{l+1}+\frac{1}{3} \leq \beta_{F}^{-}<\frac{c 3}{2},
$$

which implies $k=l=1$, i.e., $n=m$ and $v=2 n$. Since $k n+l m+u=2 v$, we have $u=v$, a contradiction with $v>u$. Hence $E$ must be a component of the horizontal branch. Without loss of generality, we assume that $E$ is on the right branch. Consider the contribution to $\beta_{F}^{-}$; we have $k=1$ and $E$ intersects with the $(-2)$-curve at the end. The dual graph of $F$ is as follows:

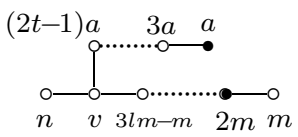

We have $v=2 n=(2 t+1) a=(3 l+2) m$, and $n+(2 t-1) a+(3 l-1) m=2 v$. It implies

$$
\frac{1}{2}+\frac{2 t-1}{2 t+1}+\frac{3 l-1}{3 l+2}=2,
$$


i.e.,

$$
\frac{2}{2 t+1}+\frac{3}{3 l+2}=\frac{1}{2}
$$

This equation has only one solution, $t=3$ and $l=4$. Now we can compute $\beta_{F}^{-}=\frac{11}{7}>\frac{3}{2}$, a contradiction.

We have proved that $s=2$ cannot occur. So $s=1$. The claim is proved.

Finally, we need to exclude the case in the claim.

$F$ has exactly two H-J branches whose end points are $(-3)$-curves. The remaining H-J branch is of type $A_{n}$ which contains $k$ vertexes. The dual graph is as follows:

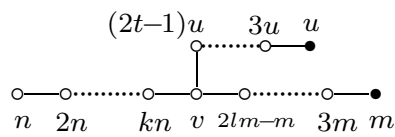

where $(2 l+1) m=(2 t+1) u=(k+1) n=v(l \leq t)$ and $\frac{2 l-1}{2 l+1}+\frac{2 t-1}{2 t+1}+\frac{k}{k+1}=2$ by Zariski's lemma, i.e., $\frac{1}{2 l+1}+\frac{1}{2 t+1}=\frac{k}{2 k+2}$. Then we have

$$
\begin{aligned}
\beta_{F}^{-} & =\frac{l}{2 l+1}+\frac{t}{2 t+1}+\frac{k}{k+1} \\
& =1-\frac{1}{2}\left(\frac{1}{2 l+1}+\frac{1}{2 t+1}\right)+\frac{k}{k+1} \\
& =1-\frac{1}{4} \cdot \frac{k}{k+1}+\frac{k}{k+1}=1+\frac{3}{4} \cdot \frac{k}{k+1}<\frac{3}{2} ;
\end{aligned}
$$

we get $k=1$. It is easy to see that the equation $\frac{1}{2 l+1}+\frac{1}{2 t+1}=\frac{1}{4}$ has no positive integral solutions $l$ and $t$. So the case in the claim is excluded. Hence $F_{\text {red }} K_{X}=2$ is impossible.

The lemma is finally proved.

Now $F$ consists of one (-3)-curve $C_{0}$ and some connected $A D E$ curves $\Gamma_{1}, \cdots, \Gamma_{r}$. Let $Z_{i}$ be the fundamental cycle supported on $\Gamma_{i}$. Then $Z_{i}^{2}=-2$. See ([5], Ch. III, $\S 3)$ for the list of $Z_{i}$.

Since $\left(C_{0}+Z_{i}\right)^{2} \leq 0,1 \leq C_{0} Z_{i} \leq 2$. If $C_{0} Z_{i}=2$, then $Z_{i}$ cannot be of type $A_{n}$. Otherwise $Z_{i}$ is reduced and $C_{0} Z_{i}=2$ implies that $F$ is not a tree. Hence $Z_{i}$ must be of types $E_{k}$ or $D_{n}$.

Lemma 5.6. If $C_{0} Z_{i}=2$ for some $i$, then $g=2$ and $F$ is of types $10 \sim 16$.

Proof.

Step 1 . There is at most one $Z_{i}$ such that $C_{0} Z_{i}=2$. Otherwise if $Z_{i}$ and $Z_{j}$ satisfy $C_{0} Z_{i}=C_{0} Z_{j}=2$, then $\left(C_{0}+Z_{1}+Z_{2}\right)^{2}=1$, a contradiction. Without loss of generality, we assume $C_{0} Z_{1}=2$ and $C_{0} Z_{i}=1$ for all $i \geq 2$.

Step 2. Suppose $r \geq 3$. One can check that

$$
\left(2 C_{0}+2 Z_{1}+Z_{2}+Z_{3}\right)^{2}=0 .
$$

Note that $F$ is simply connected and cannot be a multiple fiber (18, p. 389). So $F=2 C_{0}+2 Z_{1}+Z_{2}+Z_{3}$. Let $C_{2}$ be an irreducible component of $Z_{2}$ such that $C_{2} Z_{2}<0$. From $F C_{2}=0$ we get $C_{2} Z_{2}=-2 C_{0} C_{2}$. Since $\left(Z_{2}-C_{2}\right)^{2} \leq 0$, we have $C_{2} Z_{2} \geq-2$, so $C_{2} Z_{2}=-2$ and $\left(C_{2}-Z_{2}\right)^{2}=0$, i.e., $Z_{2}=C_{2}$ is just one $(-2)$-curve. Similarly, $Z_{3}$ is also a $(-2)$-curve. Recall that $\operatorname{supp}\left(Z_{1}\right)$ is a curve of types $D_{n}$ or $E_{k}$, and $C_{0}$ meets with $Z_{1}$ at the component $E$ with $E Z_{1}<0$. Because $\beta_{F}^{-}<\frac{5}{2}, Z_{1}$ 
cannot be of type $D_{n}$. Now one can check that the possibilities are just the fibers of types 11,12 and 13 .

Step 3. Suppose $r=2$. Let $C_{1}$ be the irreducible component of $Z_{1}$ such that $C_{1} Z_{1}<$ 0 . Since $Z_{1}$ is not a curve of type $A_{n}$, one can check from the list that $C_{1} Z_{1}=-1$. Then $\left(2 C_{0}+2 Z_{1}+Z_{2}-C_{1}\right)^{2}=-4 C_{0} C_{1}$. If $C_{0} C_{1}=0, F=2 C_{0}+2 Z_{1}+Z_{2}-C_{1}$. By Zariski's lemma, $0=F Z_{1}=-C_{1} Z_{1}$, a contradiction. So $C_{0} C_{1}=1$.

Let $C_{2}$ be an irreducible component of $Z_{2}$ such that $C_{0} C_{2}=1$. Since $C_{0} Z_{2}=1$, the multiplicity of $C_{2}$ in $Z_{2}$ is 1 . If $Z_{2} C_{2}<0$, then one can check that $Z_{2}$ is of type $A_{n}, C_{2}$ is at the end of $Z_{2}$ and $C_{2} Z_{2}=-1$. Consider $D=C_{0}+Z_{1}+Z_{2}$; one can check that $D \Gamma \leq 0$ for each irreducible $\Gamma$ of $D$, e.g., $C_{1} D=0$ and $C_{2} D=0$. $D^{2}=-1$. By Lemma 5.2, $D$ is a negative curve, a contradiction. Hence $Z_{2} C_{2}=0$.

Now we have

$$
\left(2 C_{0}+2 Z_{1}+Z_{2}+C_{2}\right)^{2}=0 .
$$

Thus $F=2 C_{0}+2 Z_{1}+Z_{2}+C_{2}$. Since $Z_{2} C_{2}=0, Z_{2}$ cannot be irreducible. There is another component $C_{3}$ of $Z_{2}$ such that $Z_{2} C_{3}<0$. Since $0=F C_{3}=Z_{2} C_{3}+C_{2} C_{3}$, we see that $Z_{2} C_{3}=-1$, and $C_{2} C_{3}=1$. Check each type of $A D E$ fundamental cycles; one find that $Z_{2}$ must be of type $D_{n}$. From $\beta_{F}^{-}<\frac{5}{2}$, we see that the dual graph of $F$ has at most four $(-2)$-curves as its end points, so $Z_{1}$ cannot be of type $D_{n}$. Now we obtain that $F$ is of types 14,15 and 16 .

Step 4. Suppose $r=1$. Let $C_{1}$ be the irreducible component of $Z_{1}$ such that $C_{0} C_{1}=1$. If $C_{1} Z_{1}<0, C_{0}+Z_{1}$ is a negative cycle by Lemma 5.2, a contradiction. So $C_{1} Z_{1}=0$. Let $C_{2}$ be another irreducible component of $Z_{1}$ such that $Z_{1} C_{2}<0$; one can check that $Z_{1} C_{2}=-1$.

If $C_{1} C_{2}=0$, then

$$
\left(2 C_{0}+2 Z_{1}+C_{1}-C_{2}\right)^{2}=0 .
$$

So $F=2 C_{0}+2 Z_{1}+C_{1}-C_{2}$. Thus $0=F C_{0}=-1-C_{0} C_{2}$, a contradiction. Hence $C_{1} C_{2}=1$. By checking each type of $A D E$ fundamental cycles, we see that $Z_{1}$ is of type $D_{n}$. Note that $C_{2}$ is unique in $Z_{1}$. We claim that $C_{1}$ is not at the end of $D_{n}$. Otherwise, by Lemma 5.2, $C_{0}+Z_{1}+C_{1}$ is a negative cycle, a contradiction. So the position of $C_{1}$ is determined. Now we easily see that $F$ is just the fiber of type 10 .

From now on we always assume that $C_{0} Z_{i}=1$ for all $i$. So $C_{0}$ meets with a component whose multiplicity in $Z_{i}$ is 1 . From Zariski's lemma, one can determine the multiplicities of the irreducible components of $Z_{i}$ in $F$ whenever the multiplicity of $C_{0}$ is determined. The following are all possible partial dual graphs of $C_{0}$ and $Z_{i}$ in $F$ :
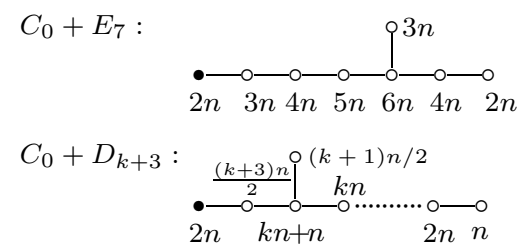

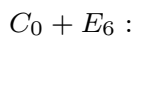

$C_{0}+D_{m}^{*}$ :
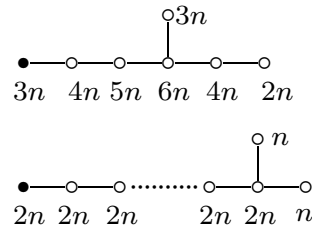

$C_{0}+A_{k+l+1}: \quad \begin{array}{ll}m+n \\ m 2 m\end{array}$ 
Recall that $F_{\text {red }}-C_{0}$ consists of $r$ connected components $\Gamma_{1}, \cdots, \Gamma_{r}$ and $\beta_{F}^{-}<\frac{5}{2}$. Let $C_{i}$ be the irreducible component of $\Gamma_{i}$ meeting with $C_{0}$, and let $n_{i}$ be the multiplicity of $C_{i}$ in $F$. Let $\beta_{i}$ be the contribution of $\Gamma_{i}$ to $\beta_{F}^{-}$. If $r \geq 2$, then we have

$$
3=\sum_{i=1}^{r} \frac{n_{i}}{n_{0}}, \quad \beta_{F}^{-}=\sum_{i=1}^{r} \beta_{i}<\frac{5}{2} .
$$

Lemma 5.7. 1) $r \leq 3$, and if $r=3$, then $F$ is the fiber of type 18 .

2) If $r=2$ and all $\Gamma_{i}$ are not of type $A_{n}$, then $F$ is of types 5,19, 20 and 22 .

3) If $r=2$ and $\Gamma_{1}$ is of type $A_{n}$, then $F$ is of types $1,2,4,6,9$ and 17.

4) If $r=1$, then $F$ is of types 3,7 and 8 .

Proof. 1) Note first that if all $\Gamma_{i}$ are of type $A_{n}$ and form some H-J branches, then by a straightforward computation we have $\beta_{F}^{-}=\sum_{i=1}^{r} \frac{n_{i}}{n_{0}}=3>\frac{5}{2}$, a contradiction. Since $\beta_{F}^{-}<\frac{5}{2}, r \leq 4$. If $r=4$, then all $\Gamma_{i}$ are of type $A_{n}$ and form H-J branches, which is impossible. Hence $r \leq 3$.

Assume $r=3$. $\beta_{F}^{-}<\frac{5}{2}$ implies $F_{\text {red }}-C_{0}$ contains two H-J branches of type $A_{n}$, say $\Gamma_{1}$ and $\Gamma_{2}$. By (5.3), we have

$$
\beta_{F}^{-}=\frac{n_{1}}{n_{0}}+\frac{n_{2}}{n_{0}}+\beta_{3}=3-\frac{n_{3}}{n_{0}}+\beta_{3} .
$$

If $\Gamma_{3}$ is of type $E_{7}, E_{6}$ or $D_{m}^{*}$, then one can check that $\beta_{F}^{-}=\frac{8}{3}, \frac{17}{6}$ and 3 respectively, which contradicts the condition $\beta_{F}^{-}<\frac{5}{2}$. If $\Gamma_{3}$ is of type $A_{k+l+1}$ as above, then $k \geq 1$ and $l \geq 1$ (since $\Gamma_{3}$ is not an H-J branch of type $A_{n}$ ). On the other hand, $\frac{n_{1}}{n_{0}} \geq \frac{1}{2}$ and $\frac{n_{2}}{n_{0}} \geq \frac{1}{2}$, so $\frac{n_{3}}{n_{0}} \leq 2 . n_{3}=(l+1) m=(k+1) n$, so $\frac{n_{3}}{n_{0}}=\frac{(k+1)(l+1)}{(k+1)+(l+1)} \leq 2(l \leq k)$; we obtain that $l=1$. Hence

$$
\beta_{F}^{-}=3+\frac{k}{k+1}+\frac{1}{2}-\frac{2(k+1)}{k+3}=\frac{5}{2}+\frac{3 k+1}{(k+1)(k+3)}>\frac{5}{2},
$$

a contradiction.

Finally, assume that $\Gamma_{3}$ is of type $D_{k+3}$ as above. $\beta_{3}=\frac{1}{2}+\frac{k}{k+1}$, so

$$
\beta_{F}^{-}=3+\frac{1}{2}+\frac{k}{k+1}-\frac{k+3}{4}<\frac{5}{2} ;
$$

we get $k \geq 5$. On the other hand, $\frac{n_{3}}{n_{0}} \leq 2$, i.e., $\frac{k+3}{4} \leq 2$ and $k \leq 5$. Hence $k=5$, $2 n_{1}=2 n_{2}=n_{0}=2 n$. Because $F$ cannot be a multiple fiber, $n=1$. This is just the fiber of type 18 .

2 ), 3) and 4) can be proved by similar calculations.

5.4. Applications. The local canonical class inequality has some interesting applications. It has been used to establish the canonical class inequality for nonsemistable fibrations. Now we give a new proof of the following well-known result.

Corollary 5.8. Let $f: X \rightarrow \mathbb{P}^{1}$ be a non-trivial fibration of genus $g \geq 1$. Then $f$ admits at least 2 singular fibers.

Proof. If $f$ is smooth, then it is trivial. Now we assume that $f$ admits only one singular fiber $F$. In this case, $f$ is isotrivial. So

$$
c_{1}^{2}(X)=-8(g-1)+c_{1}^{2}(F), \quad c_{2}(X)=-4(g-1)+c_{2}(F) .
$$

If $g \geq 2$, we proved in [16] that $c_{1}^{2}(X)+8(g-1)=K_{f}^{2} \geq 4(g-1)$. By (5.4), we have $c_{1}^{2}(F) \geq 4 g-4$, a contradiction. 
If $g=1$, then $12 \chi\left(\mathcal{O}_{X}\right)=c_{2}(X)=c_{2}(F)$. So $c_{2}(F)$ is divided by 12 . We know that $c_{2}(F)=0$, and $F=n E$ for some smooth elliptic curve $E$ and $n \geq 2$. Hence $\chi\left(\mathcal{O}_{X}\right)=0$. By the formula for the canonical class, we have $K_{X} \sim-(n+1) E$. Hence $X$ is birationally ruled, $p_{g}(X)=0$ and $q(X)=1$. The Albanese map $\alpha: X \rightarrow B$ is the ruling. Let $F^{\prime}$ be a fiber of $\alpha$. Then $2=-K_{X} F^{\prime}=(n+1) E F^{\prime} \geq$ $n+1 \geq 3$, a contradiction.

This proves that $f$ admits at least 2 singular fibers.

5.5. Chern numbers of the fibers in Theorem 1.3. In order to prove 2) of Theorem 1.2, we need to compute the Chern numbers for all 22 singular fibers in Theorem 1.3 .

\begin{tabular}{|c|c|c|c|c|c|c|c|c|c|c|c|}
\hline$F$ & 1 & 2 & 3 & 4 & 5 & 6 & 7 & 8 & 9 & 10 & 11 \\
\hline$g$ & 6 & 4 & 3 & 3 & 3 & 3 & 2 & 2 & 2 & 2 & 2 \\
\hline$c_{1}^{2}$ & $\frac{130}{7}$ & $\frac{54}{5}$ & 7 & $\frac{48}{7}$ & $\frac{98}{15}$ & $\frac{20}{3}$ & $\frac{16}{5}$ & 3 & 3 & 3 & $\frac{8}{3}$ \\
\hline$c_{2}$ & 30 & 26 & 21 & 18 & $\frac{268}{15}$ & 20 & 16 & 15 & 15 & 9 & $\frac{34}{3}$ \\
\hline$\chi$ & $\frac{85}{21}$ & $\frac{46}{15}$ & $\frac{7}{3}$ & $\frac{29}{14}$ & $\frac{61}{30}$ & $\frac{20}{9}$ & $\frac{8}{5}$ & $\frac{3}{2}$ & $\frac{3}{2}$ & 1 & $\frac{7}{6}$ \\
\hline
\end{tabular}

\begin{tabular}{|c|c|c|c|c|c|c|c|c|c|c|c|}
\hline$F$ & 12 & 13 & 14 & 15 & 16 & 17 & 18 & 19 & 20 & 21 & 22 \\
\hline$g$ & 2 & 2 & 2 & 2 & 2 & 2 & 2 & 2 & 2 & 2 & 2 \\
\hline$c_{1}^{2}$ & $\frac{11}{4}$ & $\frac{17}{6}$ & $\frac{8}{3}$ & $\frac{11}{4}$ & $\frac{17}{6}$ & $\frac{14}{5}$ & $\frac{8}{3}$ & $\frac{8}{3}$ & $\frac{8}{3}$ & $\frac{13}{5}$ & $\frac{31}{12}$ \\
\hline$c_{2}$ & $\frac{49}{4}$ & $\frac{79}{6}$ & $\frac{34}{3}$ & $\frac{49}{4}$ & $\frac{79}{6}$ & 14 & $\frac{40}{3}$ & $\frac{40}{3}$ & $\frac{52}{3}$ & 7 & $\frac{197}{12}$ \\
\hline$\chi$ & $\frac{5}{4}$ & $\frac{4}{3}$ & $\frac{7}{6}$ & $\frac{5}{4}$ & $\frac{4}{3}$ & $\frac{7}{5}$ & $\frac{4}{3}$ & $\frac{4}{3}$ & $\frac{5}{3}$ & $\frac{4}{5}$ & $\frac{19}{12}$ \\
\hline
\end{tabular}

\section{Proof of Theorem 1.4}

In this section, we will classify all singular fibers satisfying $2 c_{2}(F)-c_{1}^{2}(F)<6$.

Lemma 6.1. If $2 c_{2}(F)-c_{1}^{2}(F) \neq 0$, then $2 c_{2}(F)-c_{1}^{2}(F) \geq 3$.

Proof. We have

$$
2 c_{2}(F)-c_{1}^{2}(F)=2\left(\mu_{F}-\beta_{F}-\alpha_{F}\right)+\alpha_{F}+3 \beta_{F}^{-}-F_{\text {red }}^{2}<3 .
$$

In particular, we have $\sum_{p \in F}\left(2\left(\mu_{p}-\beta_{p}-\alpha_{p}\right)+\alpha_{p}+3 \beta_{p}^{-}\right)<3+F_{\text {red }}^{2} \leq 3$. By Lemma 3.2, 4), $F$ is a nodal curve, and so $\alpha_{F}=0$. If $F_{\text {red }}^{2}=0$, then $F=n F_{\text {red }}$ and $2 c_{2}(F)-c_{1}^{2}(F)=0$. If $F_{\text {red }}^{2} \leq-1$, then $\mu_{F}-\beta_{F}<1$. Note that if a node $p$ satisfies $\beta_{p} \neq 1$, then $\beta_{p} \leq \frac{1}{2}$, and $\mu_{p}-\beta_{p} \geq \frac{1}{2}$. Hence $\mu_{F}-\beta_{F}<1$ implies that at most one node $p$ satisfies $\beta_{p} \neq 1$. So $F=n A+m B, A$ and $B$ are reduced nodal curves and $A B=1$. By Zariski's lemma, $0=A F=n A^{2}+m A B=n A^{2}+m$; similarly, $n+m B^{2}=0$. Hence $m=n$, and $F_{\text {red }}^{2}=0$, a contradiction. This proves the lemma.

Proof of Theorem 1.4. We can assume that $2 c_{2}(F)-c_{1}^{2}(F) \geq 3$ :

$$
3 \leq 2\left(\mu_{F}-\beta_{F}-\alpha_{F}\right)+\alpha_{F}+3 \beta_{F}^{-}-F_{\text {red }}^{2}<6 .
$$


In particular, we have $\sum_{p \in F}\left(2\left(\mu_{p}-\beta_{p}-\alpha_{p}\right)+\alpha_{p}+3 \beta_{p}^{-}\right)<6+F_{\text {red }}^{2} \leq 6$. By Lemma 3.2, 4), $F$ admits at most one singular point $p$ which is not a node, and $p$ is of type $A_{2}, A_{3}$ or $D_{4}$.

If $F_{\text {red }}^{2}=0$, then $F=n F_{\text {red }}$. Then one can compute all the local invariants directly, and we get the cases 2$) \sim 5$ ). In what follows we assume that $F_{\text {red }}^{2} \leq-1$. So $\sum_{p \in F}\left(2\left(\mu_{p}-\beta_{p}-\alpha_{p}\right)+\alpha_{p}+3 \beta_{p}^{-}\right)<5$, and $p$ is at worst of $A_{3}$.

Let $s$ be the number of nodes in $F_{\text {red }}$ satisfying $\beta_{q}<1$. For such a node $q$, the two components of $F$ at $q$ have distinct multiplicities. So $\beta_{q} \leq \frac{1}{2}$ and $\mu_{q}-\beta_{q} \geq \frac{1}{2}$.

If the non-nodal singular point $p$ exists, then $p$ is of type $A_{2}$ or $A_{3}$. Also, $2\left(\mu_{p}-\beta_{p}-\alpha_{p}\right)+\alpha_{p}+3 \beta_{p}^{-}$is at least $\frac{7}{2}$ if $p$ is of type $A_{2}$ or $A_{3}$, so we get $\frac{7}{2}+s<5$, i.e., $s \leq 1$. As in the proof of the previous lemma, $s=1$ is impossible by Zariski's lemma. So $s=0$, i.e., the multiplicities of the two local branches of any node are the same. Hence $p$ is of type $A_{3}$.

From Zariski's lemma, one has a decomposition $F=n(A+2 B)$, where $A$ and $B$ are connected reduced nodal curves and smooth at $p, A \cap B=\{p\}, A^{2}=-4$, $B^{2}=-1, A B=2$. We get case 8$)$.

From now on we always assume $F_{\text {red }}$ is a nodal curve. By assumption, $s \neq 0$. As in the proof of the previous lemma, $s=1$ is also impossible. So $2 \leq s<6+F_{\text {red }}^{2} \leq 5$ by (6.2), i.e., $2 \leq s \leq 4$.

Let $F=\gamma_{1} \Gamma_{1}+\cdots+\gamma_{r} \Gamma_{r}, r \geq 2$, where the $\Gamma_{i}$ 's are reduced with $\Gamma_{i}^{2}=-e_{i} \leq-1$ (not necessarily irreducible) and have no pairwise common components. $\gamma_{1}, \cdots, \gamma_{r}$ are pairwise distinct. Then we have

$$
r-1 \leq \sum_{i<j} \Gamma_{i} \Gamma_{j}=s, \quad F_{\text {red }}^{2}=2 s-e_{1}-\cdots-e_{r}
$$

If $r-1=s$, then $\Gamma_{1}, \cdots, \Gamma_{r}$ form a chain. Assume that this is a chain such as the one before Lemma 2.6 where $\gamma_{0}=\gamma_{r+1}=0$. So the linear equation (2.1) holds true. Since $\gamma_{0}=0$, we can see from the equation that $\gamma_{1}$ divides $\gamma_{i}$ for any $i$. Symmetrically, from $\gamma_{r+1}=0$, we know that $\gamma_{r}$ divides $\gamma_{i}$ for all $i$. So $\gamma_{1}=\gamma_{r}$, which contradict our assumption. So $r \leq s$.

Suppose $s=2$. Then $r=2$. Now one can prove that $F=n A+2 n B, A B=2$, $A^{2}=-4$ and $B^{2}=-1$. We get cases 6 ) and 7).

Suppose $s=3$. Then $F_{\text {red }}^{2} \geq-2$ and $r=2$ or 3 .

If $r=2$, then one can prove that $F=\gamma_{1} \Gamma_{1}+3 \gamma_{1} \Gamma_{2}, \Gamma_{1} \Gamma_{2}=3$. Hence $\mu_{F}-\beta_{F}=$ $3-1=2$. Now we have $2\left(\mu_{F}-\beta_{F}\right) \geq 6+F_{\text {red }}^{2}$, a contradiction.

If $r=3$ and $\Gamma_{1} \Gamma_{2}=\Gamma_{2} \Gamma_{3}=\Gamma_{3} \Gamma_{1}=1$, then one can prove that $F=\gamma_{1} \Gamma_{1}+$ $3 \gamma_{1} \Gamma_{2}+2 \gamma_{1} \Gamma_{3}$. Hence $\mu_{F}-\beta_{F}=3-1=2$ and $2\left(\mu_{F}-\beta_{F}\right) \geq 6+F_{\text {red }}^{2}$, a contradiction.

If $r=3, \Gamma_{1} \Gamma_{2}=2, \Gamma_{2} \Gamma_{3}=1$ and $\Gamma_{3} \Gamma_{1}=0$, then we have $-e_{1} \gamma_{1}+2 \gamma_{2}=0$, $2 \gamma_{1}-e_{2} \gamma_{2}+\gamma_{3}=0$ and $\gamma_{2}-e_{3} \gamma_{3}=0$. Since $\gamma_{2} \neq \gamma_{3}$, we have $e_{3} \geq 2$. We obtain that $e_{1}=e_{3}\left(e_{1} e_{2}-4\right)$, which implies $e_{2}=1$. $e_{1}=e_{3}=5$, or $e_{1}=6$ and $e_{3}=3$, or $e_{1}=8$ and $e_{3}=2$. Hence $F_{\text {red }}^{2} \leq-4$, a contradiction.

Suppose $s=4$. Then $F_{\text {red }}^{2}=-1$. Let $F=\sum_{i=1}^{k} n_{i} C_{i}$, where $C_{i}$ is irreducible. Let $q_{1}, \cdots, q_{4}$ be the nodes satisfying $\frac{1}{2} \geq \beta_{q_{1}} \geq \cdots \geq \beta_{q_{4}}$. By (6.2), we have

$$
2 \sum_{k=1}^{4}\left(\mu_{q_{k}}-\beta_{q_{k}}\right)<5,
$$


which implies $\beta_{q_{1}}=\beta_{q_{2}}=\frac{1}{2}$. Let $C_{i_{1}}$ and $C_{i_{2}}$ be the components passing through $q_{i}(i \leq 4)$ and let $n_{i 1} \leq n_{i 2}$. From $\beta_{q_{1}}=\beta_{q_{2}}=\frac{1}{2}$, we have $n_{i 2}=2 n_{i 1}$ for $i=1$ and 2 , and thus $\chi\left(n_{11}, n_{12}\right)=\chi\left(n_{21}, n_{22}\right)=0$.

From the proof of Theorem 4.1, we have

$$
\sum_{k \leq 4}\left(\mu_{q_{k}}-\beta_{q_{k}}\right)+F_{\text {red }}^{2}=-12 \sum_{i \leq 4} \chi\left(n_{i 1}, n_{i 2}\right) .
$$

Since $F_{\text {red }}^{2}=-1$ and $\beta_{q_{1}}=\beta_{q_{2}}=\frac{1}{2}$,

$$
\mu_{q_{3}}-\beta_{q_{3}}+\mu_{q_{4}}-\beta_{q_{4}}=6-\left(\frac{n_{31}}{n_{32}}+\frac{n_{32}}{n_{31}}\right)-\left(\frac{n_{41}}{n_{42}}+\frac{n_{42}}{n_{41}}\right)-\beta_{q_{3}}-\beta_{q_{4}},
$$

i.e.,

$$
4=\left(\frac{n_{31}}{n_{32}}+\frac{n_{32}}{n_{31}}\right)+\left(\frac{n_{41}}{n_{42}}+\frac{n_{42}}{n_{41}}\right) \geq 2+2=4 .
$$

Thus $n_{31}=n_{32}$ and $n_{41}=n_{42}$, so $\beta_{q_{3}}=\beta_{q_{4}}=1$, a contradiction.

Up to now we have completed the proof.

Corollary 6.2. If the semistable model of $F$ is smooth and $F$ is not the multiple of a smooth curve, then $2 c_{2}(F)-c_{1}^{2}(F) \geq 6$.

Corollary $6.3([10])$. If $f: X \rightarrow C$ is an isotrivial family of curves, then $K_{X}^{2} \neq$ $8 \chi\left(\mathcal{O}_{X}\right)-1$.

Proof. In this case, the modular invariants of $f$ are zero. Hence

$$
2 c_{2}(X)-c_{1}^{2}(X)=\sum_{i}\left(2 c_{2}\left(F_{i}\right)-c_{1}^{2}\left(F_{i}\right)\right) \geq 0 .
$$

Suppose $K_{X}^{2} \neq 8 \chi\left(\mathcal{O}_{X}\right)$, i.e., $2 c_{2}(X) \neq c_{1}^{2}(X)$. Then at least one singular fiber satisfies the condition of Corollary 6.2. Hence $2 c_{2}(X)-c_{1}^{2}(X) \geq 6$ and , equivalently, $K_{X}^{2} \leq 8 \chi\left(\mathcal{O}_{X}\right)-2$.

Corollary 6.4. If $F$ is not semistable, then $c_{2}(F) \geq \frac{11}{6}$ and $\chi_{F} \geq \frac{1}{6}$. One of the equalities holds if and only if $F$ is a reduced curve with one ordinary cusp and some nodes.

Proof. If $2 c_{2}(F)-c_{1}^{2}(F) \geq 6$, equivalently, $8 \chi_{F}-c_{1}^{2}(F) \geq 2$, then $c_{2}(F)>3$ and $\chi_{F}>\frac{1}{4}$. So we can assume that $2 c_{2}(F)-c_{1}^{2}(F)<6$. By Theorem 1.4, we have 8 types of singular fibers. We see that only the type 2) fiber with $N_{F}=0$ has the minimal $c_{2}(F)$ and $\chi_{F}$. This proves the corollary.

\section{Questions.}

1) What is the upper bound of $c_{2}(F)$ ? We conjecture $c_{2}(F) \leq \frac{55 g}{6}$.

2) Is $\frac{1}{6}$ the lower bound of $c_{1}^{2}(F)$ for a minimal non-semistable fiber $F$ ?

3) Is the inequality $c_{1}^{2}(F) \geq \chi_{F}$ true for any minimal singular fiber $F$ ? (If $F$ is a singular fiber in an isotrivial family, then one can easily prove that $c_{1}^{2}(F) \geq$ $\left.\frac{4(g-1)}{g} \chi_{F} \cdot\right)$

\section{ACKNOWLEDGEMENTS}

The authors would like to thank the referees for valuable suggestions for the correction of the original manuscript. 


\section{REFERENCES}

[1] M. Artin: On isolated rational singularities of surfaces, Amer. J. Math., 88 (1966), 129-136. MR0199191 (33:7340)

[2] T. Ashikaga, M. Ishizaka: Classification of degenerations of curves of genus three via Matsumoto-Montesino's theorem, Tohoku Math. J., 54 (2002), 195-226. MR.1904949 (2003g:14011)

[3] T. Ashikaga and K. Konno: Global and local properties of pencils of algebraic curves, Algebraic Geometry 2000, Azumino, Advanced Studies in Pure Mathematics, 36 (2000), 1-49. MR:1971511 (2004f:14051)

[4] A. Beauville: L'inégalité pour les surfaces de type générale, Appendix to: O. Debarre, Inégalités numériques pour les surfaces de type general, Bull. Soc. Math. de France, 110 (1982), 319-346. MR688038 (84f:14026)

[5] W. Barth, C. Peter, A. Van de ven: Compact complex surfaces, Berlin, Heidelberg, New York: Springer, 1984. MR749574 (86c:32026)

[6] S. Iitaka: Master degree thesis, University of Tokoyo (1967).

[7] K. Kodaira: On compact analytic surfaces, III, Ann. of Math. 78 (1963), no. 1, 1-40. MR0184257 (32:1730)

[8] Y. Namikawa, K. Ueno: On fibers in families of curves of genus two. I, Algebraic Geometry and Commutative Algebra, in honor of Y. Akizuki, Kinokuniya, Tokoyo (1973), 297-371. MR0384794 (52:5667a)

[9] A. P. Ogg: On pencils of curves of genus two, Topology, 5 (1966), 355-362. MR0201437 $(34: 1321)$

[10] F. Polizzi: Numerical properties of isotrivial fibrations, Geom. Dedicata, 147 (2010), 323-355. MR.2660583(2011g:14023)

[11] S.-L. Tan: On the base changes of pencils of curves, I, Manus. Math., 84 (1994), 225-244. MR.1291119 (95h:14006)

[12] S.-L. Tan: The minimal number of singular fibers of a semistable curve over $\mathbb{P}^{1}$, J. Algebraic Geom., 4 (1995), 591-596. MR.1325793 (96e:14038)

[13] S.-L. Tan: On the base changes of pencils of curves. II, Math. Z., 222 (1996), 655-676. MR 1406272 (98b:14004)

[14] S.-L. Tan: On the slopes of the moduli spaces of curves, Intern. J. of Math., 9 (1998), 119-127. MR $1612259(99 \mathrm{k}: 14042)$

[15] S.-L. Tan: Chern numbers of a singular fiber, modular invariants and isotrivial families of curves, Acta Math. Viet., 35 (2010), no. 1, 159-172. MR2642167 (2011g:14069)

[16] S.-L. Tan, Y.-P. Tu, and A.-G. Zamora: On complex surfaces with 5 or 6 semistable singular fibers over $\mathbb{P}^{1}$, Math. Zeit., 249 (2005), 427-438. MR2115452 (2006f:14008)

[17] K. Uematsu: Numerical classification of singular fibers in genus 3 pencils, J. Math. Kyoto Univ. 39-4 (1999), 763-782. MR.1740203 (2001c:14014)

[18] G. Xiao: On the stable reduction of pencils of curves, Math. Z., 203 (1990), 379-389. MR.1038707 (91e:14022)

[19] G. Xiao: The fibrations of algbraic surfaces, Shanghai Scientific \& Technical Publishers, 1992 (in Chinese).

Department of Mathematics, East China Normal University, Dongchuan RD 500, Shanghai 200241, People's Republic of China

E-mail address: jlu@math.ecnu.edu.cn

Department of Mathematics, East China Normal University, Dongchuan RD 500, Shanghai 200241, People's Republic of China

E-mail address: sltan@math.ecnu.edu.cn 